\title{
The Market for Conflicted Advice*
}

\author{
Briana Chang $^{\dagger} \quad$ Martin Szydlowski ${ }^{\ddagger}$
}

January 13, 2017

\begin{abstract}
We study decentralized markets in which advisers have conflicts of interest and compete for customers via information provision. We show that competition partially disciplines conflicted advisers. The equilibrium features information dispersion and sorting of heterogeneous customers and advisers: advisers with expertise in more information sensitive assets attract less informed customers, provide worse information, and earn higher profits. We apply our framework to the market for financial advice and establish new insights: while the conflicted fee structure affects asset return, it is irrelevant for the welfare of consumers. It is the underlying distribution of financial literacy that determines the consumers' welfare.
\end{abstract}

Keywords: Information Provision, Conflicted Advice, Two-Sided Matching, Sorting JEL Codes: Cr8, D83, G11, G23

${ }^{*}$ We would like to thank Dean Corbae, Mark Egan, Pablo Kurlat, Rasmus Lentz, Gregor Matvos, Christine Parlour, Erwan Quintin, Mark Ready, Shouyong Shi and Randy Wright for their useful discussions, as well as participants at the Wisconsin School of Business, 8th Annual Conference on Money, Banking and Asset Markets, Fall 2016 Midwest Economic Theory Conference, 2016 Summer Workshop on Money, Banking, Payments and Finance, University of Wisconsin, University of Minnesota for helpful comments.

${ }^{\dagger}$ University of Wisconsin-Madison. Email: briana.chang@wisc.edu

${ }^{\ddagger}$ University of Minnesota. Email: szydl002@umn.edu. 


\section{Introduction}

Providing advice is an important function of intermediaries. The compensation structure of intermediaries, however, often leads to conflicts of interest. For example, broker-dealer firms are compensated by commissions and fund distribution fees, and realtors receive fees only if they close a deal. As a result, a key subject of interest for regulators and in the empirical literature has been how these misaligned incentives affect customers. ${ }^{1}$ Yet, how markets for conflicted advice function and their consequences for investors remain unknown.

In this paper, we study information provision in decentralized markets in which advisers are subject to conflicts of interest. Advisers (brokers or realtors) are compensated only if they successfully convince their customers to take an action (buy the asset). Taking these misaligned incentives as given, we analyze to what extent competition can discipline advisers and generate valuable information for customers.

Advisers differ in their expertise and compete for customers by their information policies. Specifically, each adviser is perfectly informed about one particular type of asset, and assets differ in their information sensitivity. Hence, an adviser with expertise in a more informationsensitive asset effectively has more valuable knowledge. Customers, who differ in the quality of their ex-ante information, understand the misaligned incentives of advisers and choose their advisers optimally. After choosing an advisor, the customer receives the information, rationally updates his beliefs, and makes his investment decision.

In order to attract a particular customer, an adviser must provide sufficiently valuable information so that the customer does not prefer to match with someone else. Each adviser is subject to a capacity constraint in the sense that they can at most match only with one customer and vice versa. Thus, the environment can be understood as a matching model with two-sided heterogeneity, where information provision determines the gains for both

\footnotetext{
${ }^{1}$ Financial advice especially has received much interest in the empirical literature, which shows that financial advisers drive customers to chase returns (Linnainmaa et al. (2015)), steer them towards high-fee, actively managed investments (Mullainathan et al. (2012)), and recommend unsuitable products when they earn high commissions (Anagol et al. (2013)). As a result, portfolios of advised customers underperform (e.g. Bergstresser et al. (2009), Chalmers and Reuter (2010) and Hoechle et al. (2013)). This has drawn the attention of regulators (CEA (2015)), who have found a substantial welfare loss from conflicted advice in the United States. As a result, the department of labor has instituted a new rule holding financial advisers to the fiduciary duty standard. Other countries have enacted a variety of measures, such as banning payments from product providers to advisers (Australia, Netherlands, UK) or mandating disclosures about conflicts of interest (Canada, Germany).
} 
sides. The distribution of information quality, the value of information to customers, the profits of advisers, and the sorting patterns are thus all determined competitively.

Intuitively, since all advisers have incentives to deceive customers, they prefer to attract the least-informed ones. This drives up the value of information received by these customers. We show that, in equilibrium, the adviser with the most valuable knowledge attracts the least-informed customer. That is, the equilibrium features negative assortative matching.

As a result, less-informed customers receive less valuable information than better-informed customers, while still gaining more information than better-informed customers relative to deciding on their own. This also implies that such customers' matching advisers - those with more valuable knowledge - obtain a higher profit. Thus, competition under heterogeneity leads to different rents for different agents. Information quality is dispersed, and advisers generally provide partial disclosure.

Our framework provides a direct link between information quality and the distribution of customers' informedness and advisers' expertise. We show that when other customers in the economy become better informed, less-informed customers benefit and receive better information, even though their own level of informedness does not change.

Conceptually, the economics behind this result are analogous to the standard assignment model with price competition. In our framework, advisers see customers who are less informed as more valuable matches. The exact quality of information a customer receives in equilibrium is pinned down such that his matching adviser would not profit from attracting his next-best competitor, a customer who is slightly better informed than him. Hence, when other customers become better-informed, meaning that his competitors become less attractive from the viewpoint of advisers, the customer becomes relatively scarce and thus receives a higher rent in the form of better information.

A fundamental difference from the standard model with price competition is that the value of information is not perfectly transferable. Hence, technically, our environment falls into the class of matching problems with imperfectly transferable utilities. This suggests that the decentralized outcome may not maximize aggregate surplus. Indeed, we show that information provision is always distorted. Importantly, the equilibrium may provide both too little and too much information compared to efficient outcome.

The existence of distortion further highlights the cost of advisers' compensation structures. Specifically, our main model takes the advisers' compensation structure as given from 
the viewpoint of customers, and side payment is disallowed. This aims to capture the reality that, in practice, brokers are compensated by distribution fees set by fund issuers and do not charge a separate fee for their information service. ${ }^{2}$ As shown in Section 6.1, if fees were set competitively (e.g., if advisers could commit to refunding $12 \mathrm{~b}-1$ fees to investors), the environment could then be solved as the standard matching model with transferal utilities, which always guarantees the efficient outcome.

Despite allowing for a general message space, we provide a closed form characterization of the optimal policy. With this tractable framework, we further establish two new insights on how conflicted payments affect customers. First of all, counterintuitively, we show that the higher the advisers fees, the better the information received by customers. This is because information and fees are substitutes from the viewpoints of customers. With competition, an adviser must provide better information to the customer in order to compensate for the higher fee.

Second, we establish an equivalence of consumers' utilities under information v.s. price competition. That is, even though information is distorted in our environment, consumers' utilities generally are not. Specifically, as long as advisers are relatively scarce (i.e. they are on the short side of market), we show that all customers receive the same utilities across the two settings. That is, customers will be indifferent between getting a better information with a higher fee and getting distorted information.

Our results thus have important policy implication in the market of financial advice. When regulators limit the advisers ability to receive kickbacks, in equilibrium advisers respond by charging a higher fee. Our irrelevance result suggests that this effect balances out perfectly. Thus, while regulation aimed at conflicted payments can improve information, it, contrary to the conventional wisdom, does not actually increase consumer welfare.

Related Literature Our paper is the first to study information provision in a model of decentralized competition with heterogeneity. Methodologically, our work is built on

\footnotetext{
${ }^{2}$ See, for example, Understanding Your brokerage and Investment Advisory Relationships by Morgan Stanley (Dec 2014): "In addition to taking your orders, executing your trades and providing custody services, we also provide investor education, investment research, financial tools and professional, personalized information about financial products and services, including recommendations to our brokerage clients about whether to buy, sell or hold securities. We do not charge a separate fee for these services because these services are part of, or "incidental to," our brokerage services.
} 
the Bayesian Persuasion approach (put forth by Kamenica and Gentzkow (2011)) 3 $^{3}$ and the literature on matching markets (initiated by Becker (1973), Shapley and Shubik (1971)). ${ }^{4}$ This allows us to derive the adviser's optimal information policy without any restrictions on the messages, and to characterize the equilibrium information value for each type of customer, taking into account what other advisers offer. We are then able to establish new insights on how the underlying distribution of customers' informedness affect the equilibrium value of information. In other words, how the distribution of financial literacy affects consumers with different sophistication. This is of first-order importance for policy, since generally, less sophisticated customers are perceived as being more at risk in such markets.

Within the Bayesian Persuasion framework, other papers are studying the effects of competition, although none feature heterogeneity and sorting, which is central to our analysis. Gentzkow and Kamenica (2011) study a model where senders whose preferences differ from those a single receiver can simultaneously disclose information. They show that adding more senders or making their preferences less aligned improves the quality of information in equilibrium. ${ }^{5}$ Au and Kawai (2015) study a game with one receiver and multiple senders with possibly different priors and characterize how equilibrium information changes with each sender's prior. Unlike these two papers, we assume that a customer only observes the information from the adviser he matches with. This exclusivity of information provision is precisely what allows us to study sorting and characterize how different customers choose different advisers. Also, since these papers feature only a single receiver, they cannot generate information dispersion, i.e. different customers receiving better or worse information.

Board and Lu (2015) study a random search model in which sellers compete via persuasion. Like us, they take the conflict of interest as given and leave any underlying compensation structure or price setting unmodeled. The main tradeoff in their paper is whether a seller should provide information to discourage the buyer from continuing to search, which will depend on the value of information offered by other sellers in equilibrium. In our model, each customer can freely choose whom to match with (i.e., matching is frictionless). In or-

\footnotetext{
${ }^{3}$ Kamenica and Gentzkow (2011) analyzes the environment with one single sender with monopoly power and one receiver.

${ }^{4}$ Most works in this literature analyzes matching patterns with transferable utilities, with few exceptions: Legros and Newman (2007) provides a general sorting condition in an environment with imperfect transferable utilities. Chiappori and Reny (2006) studies a risk-sharing problem in a matching model.

${ }^{5}$ However, Li and Norman (2015) show that if senders reveal information sequentially, the receiver can be worse off.
} 
der to attract a particular customer, an adviser must provide him information value that is weakly higher than he could have obtained from other advisers. The continuation value in their model thus shares a similar spirit as our equilibrium utilities. The special case of ours where advisers are homogeneous and they are on the long side, our model would yield full disclosure, which is similar to the case of vanishing search costs in their framework.

Outside of Bayesian Persuasion, Ostrovsky and Schwarz (2010) studies the school's optimal disclosure of students' abilities in order to maximize their average job placement, taking into account that the job market features assortative matching. We share similar conflicts of interest in the sense that advisers want to maximize customers' purchasing rates. The focus in Ostrovsky's paper, however, is how one information provider affects the matching patterns between students (assets) and jobs (customers). The matching in our model, on the other hand, is between different information providers and customers.

In a series of papers by Inderst and Ottaviani, advisers must exert effort to find customers and can choose whether to recommend a product to them. Manufacturers of goods offer incentive contracts to advisers, which cannot guarantee that advisers make the recommendation that best fits the customer. Inderst and Ottaviani (2009) characterizes a monopoly problem and Inderst and Ottaviani (2012a) extend the setting to two competing manufacturers. Both papers characterize adviser fees and information provision in equilibrium, but because they do not feature any heterogeneity, they remain silent on the central questions of our paper. ${ }^{6}$ Inderst and Ottaviani (2012b) extends the monopoly case to two consumer types: naive ones who always believe advice and rational ones who understand adviser incentives. In our model, customers have different ex-ante information, but they are all fully rational. Patterns which may be interpreted as exploitation are actually the outcome of competition and policies targeting the fees of financial advisers have no effect on customer utility. Thus, our paper offers a contrasting view to their results.

\footnotetext{
${ }^{6}$ Also, in their setting, advisers are restricted to give customers a binary recommendation. A priori, it is not clear whether this is without loss of generality. Using Bayesian persuasion, our paper makes no such restrictions ex-ante. Advisers in our model can use any arbitrary information policy.
} 


\section{$2 \quad$ Model}

Customers There is a mass of heterogeneous customers who are different in terms of their ex-ante information quality. They are indexed by type $b \in B \equiv[\underline{b}, \bar{b}]$, which is observable with $0 \leq \underline{b}<\bar{b}<1$. Let $Q(b)$ denote the measure of customers with types weakly below $b$ and let $Q(\bar{b})$ denote the overall measure of customers. $Q(b)$ admits a differentiable density $q(b)$. All customers have access to a set of assets $L \equiv[\underline{l}, \bar{l}]$, which is indexed by $l$. The payoff of each asset $l$ is determined by the asset specific random variable $s \in[0,1]$, and is given by

$$
y(s, l)=\left\{\begin{array}{clc}
r & \text { if } & s \geq \lambda \\
-l & \text { if } & s<\lambda
\end{array}\right.
$$

where $s$ is distributed with strictly positive and continuous pdf $f(s)$. Thus, the asset gives a positive payoff $r>0$ only if $s \geq \lambda$. Otherwise, it results in a loss $l>0$. This assumption implies that if a customer perfectly observes the state $s$, he invests if only if $s \geq \lambda$.

Each customer $b$ can potentially invest in all assets and receives a private signal about each asset's quality, $x_{l}(s, b)=\mathbb{1}\{s \geq b \lambda\}$. Hence, by construction, if an asset is good $(s \geq \lambda)$, customers always get a positive signal $(x=1)$. However, there are false positives if $b \lambda \leq s<\lambda$. A customer with higher $b$ is thus more informed, since his signal has a lower probability of false positives.

Likewise, an asset with higher $l$ is more information sensitive: information reduces the likelihood of false positives, which is more valuable whenever $l$ is higher.

Advisers There is a unit mass of heterogeneous advisers on the other side of the market. Each adviser has expertise in one particular type of asset $l$ and is perfectly informed about the realized state $s$ of this asset. Since this is the only dimension in which advisers differ, we use $l$ to denote the type of an adviser. The distribution of adviser types is given by measure $G(l)$, which has differentiable density $g(l)$ and domain $L$.

The two-sided heterogeneity in our model captures two important dimensions of the market for information: customers (the demand side) differ in their ex-ante information and hence their needs for information; advisers (the supply side) differ in the value of the information they can provide, since an adviser who has expertise in a more information sensitive asset (i.e. a higher $l$ ) effectively has more valuable information. 


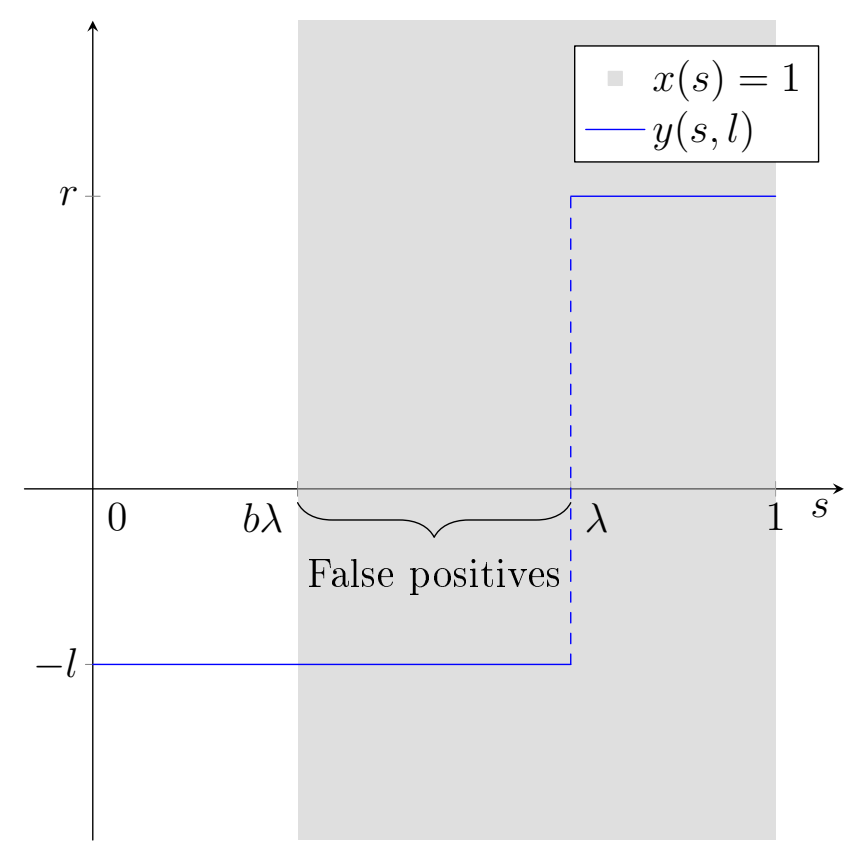

Figure 1: Payoff and Information

To capture the conflict of interest between advisers and customers, we assume that an adviser receives a positive payoff $\alpha>0$ whenever a customer invests after matching with her, independently of the realized state. This payoff captures a of conflict of interest that makes advisers have an incentive to persuade customers to invest, despite them not benefiting from it.

In the context of financial advisers, this payoff represents a commission paid by customers. Specifically, in the case of mutual funds, customers pay additional distribution fees (e.g., loads and 12b-1 fees) when they buy funds through brokers or advisors, instead of buying fund directly. ${ }^{7}$ More generally, for doctors, it may represent a kickback received by a pharmaceutical company, the cost of which is not directly passed on to the customer, ${ }^{8}$ while for lawyers it may represent the reputational gain of fighting a large trial. We consequently

\footnotetext{
${ }^{7}$ For example, Bergstresser et al. (2009) estimates that broker-channel mutual fund consumers may have paid as much as $\$ 3.6$ billion in front end loads in $2002, \$ 2.8$ billion in back-end loads and another $\$ 8.8$ billion in 12b-1 fees, in additional to the investment management fees.

${ }^{8}$ For example, if insurance covers the cost of brand medication and a doctor receives a kickback from the manufacturer which is priced into the cost of the medicine, the patient does not bear this cost when making his decision.
} 
allow the payoff to have two components: $\alpha=\alpha_{c}+\alpha_{0}$, where $\alpha_{c} \geq 0$ represents the portion paid by customers, and $\alpha_{0} \geq 0$ represents the portion that is not.

Market for advice We consider a decentralized market for advice, where different advisers compete for different customers via information provision. Specifically, we assume that each adviser can only serve one customer, and each customer can only obtain advice from one adviser (i.e., information about one type of asset $l$ ). To model the information provision of advisers, we assume that each adviser can post (and commit to) an information policy before the state is realized. The information policy consists of a signal $\sigma_{b}:[0,1] \rightarrow \Delta(\mathbb{M})$ that maps the state into a distribution over messages $m \in \mathbb{M} .^{9}$

Given the posted information policies, customers make two choices. The first decision is to choose from which adviser to seek advice. This is the matching decision. The second one is whether to invest. After the match is formed, the customer observes first his private signal $x_{l}$ for asset $l$ and then the message $m$ sent by the adviser. He then uses Bayes rule to form a posterior belief $\mu \in \Delta([0,1])$ about the state of asset $l$, conditional on both $x$ and $m$, and decides whether or not to invest. If he decides to invest, he buys the asset $l$ through the adviser $l$ and the adviser receives payoff $\alpha$.

For assets about which he does not receive advice, each customer makes his investment decision based on his own private signal. In other words, we assume that all investors can buy fund directly; hence, whether an customer purchases through advisers or not is an endogenous outcome. ${ }^{10}$ We use $u^{0}(b, l)$ to denote the expected value of customer $b$ for trading asset $l$ by himself, which is given by

$$
\begin{aligned}
u^{0}(b, l) & \equiv(1-F(b \lambda))\left(\frac{1-F(\lambda)}{1-F(b \lambda)} r-\left(1-\frac{1-F(\lambda)}{1-F(b \lambda)}\right) l\right) \\
& =(1-F(\lambda)) r-(F(\lambda)-F(b \lambda)) l .
\end{aligned}
$$

In Equation (2), with probability $1-F(b \lambda)$, the customer's private signal is high and he chooses to invest. His expected payoff is the term in brackets. The first expression is the probability that $s \geq \lambda$ conditional on $s \geq b \lambda$ times payoff $r$. The second term is the

\footnotetext{
${ }^{9}$ The particular message space $\mathbb{M}$ is irrelevant, as long as it has more than two elements. This is because the customer's action (invest or don't invest) is binary. See Kamenica and Gentzkow (2011), Prop. 1.

${ }^{10}$ In Appendix B, we consider the case where advisers act as gatekeepers for comparison.
} 


\begin{tabular}{lllll} 
& & & \\
\hline Adviser & Match is & $s$ realizes & Customer & Investment \\
posts in- & formed & Customer & observes ad- & decision $i(\mu)$ \\
formation & & observes & viser's signal & \\
policy $\sigma$ & & $x(s)$ & &
\end{tabular}

Figure 2: Timeline

probability that $s<\lambda$ conditional on $s \geq b \lambda$ times the loss $l$.

Recall that, when matching with an adviser, a customer needs to pay $\alpha_{c}$ whenever he chooses to invest. We can thus interpret parameter $\alpha_{c}$ as the additional cost if a customer chooses to invest through an adviser instead doing so on his own. To make our information market more interesting, we focus on the following parameter restrictions throughout the paper.

Assumption. $(A 1)\left(\frac{1-F(\lambda)}{1-F(\underline{b} \lambda)} r-\left(1-\frac{1-F(\lambda)}{1-F(\underline{b} \lambda)}\right) \bar{l}\right)>\alpha_{c}$. (A2) $\frac{F(\lambda)-F(\bar{b} \lambda)}{1-F(\lambda)} \underline{l}>\alpha_{c}$

Assumption (A1) guarantees that the asset is sufficiently valuable so that, after the cost $\alpha_{c}$, all customers will invest even without receiving further information from advisers. This allows us to simplify our exposition. Assumption (A2) guarantees that all advisers can provide positive value to all customers, so that all matches are potentially profitable. Under these two assumptions, if we had a monopolistic adviser, she would choose an information policy which extracts all value from customers and leaves them with their autarky value $u^{0}(b, l)$. This highlights how our results are driven by competition.

Figure 2 summarizes the timeline of our setup.

Equilibrium Our equilibrium concept is the core of the assignment game: it requires that advisers are matched to customers in a stable way. That is, any competitive equilibrium must satisfy no surplus condition: if any two agents agree to match, they cannot be better off by matching with others or using different information policies.

Denote $v(b, l, u)$ as the utility of adviser $l$, conditional on providing a customer $b$ with 
additional information value of $u$, which is given by:

$$
\begin{aligned}
& v(b, l, \bar{u})=\max _{\sigma} \tilde{V}(b, l, \sigma) \\
& \tilde{U}(b, l, \sigma)-u^{0}(b, l) \geq \bar{u}
\end{aligned}
$$

That is, by choosing an optimal policy $\sigma, v(b, l, \bar{u})$ is the payoff to an adviser conditional on leaving customer $b$ with gain $\bar{u}$. This function represents the utility possibility frontier, and in equilibrium, agents will always achieve an allocation on this frontier. Each customer can invest on his own, regardless of which adviser to chooses. Hence, he will choose the adviser who provides him the most valuable information, which is the difference between the expected value under policy $\sigma, \tilde{U}(b, l, \sigma)$, and the expected value of self-directed trade, $u^{0}(b, l)$. We let $u(b, l, \bar{v})$ denote the maximum gain to customer $b$ if he matches with an adviser $l$, who receives an utility of $\bar{v}$ within the match.

The equilibrium features of a payoff function for each agent, $U(b)$ and $V(l)$. Each agent takes the equilibrium utility of others as given and decides who to match with. We let $H(b, l)$ denote the measure of advisers below $l$ that match to customers with type below $b$ on the product of $\{B \cup \emptyset\} \times\{L \cup \emptyset\}$. Let $H^{A}(l)$ and $H^{C}(b)$ denote the marginals of this distribution, respectively. That is, $H^{A}(l) \equiv H(\bar{b}, l)$ denotes the measure of advisers with type below $l$ and $H^{C}(b) \equiv H(b, \bar{l})$ denotes the measure of customers with type below $b$. The matching is feasible if and only if the marginals coincide with the distribution of advisers and customers:

$$
H^{C}(b)=Q(b) H^{A}(l)=G(l)
$$

This marginal condition is often called the market clearing condition.

Definition 1. An equilibrium consists of matching decisions $H(b, l)$ and payoff functions $U: B \rightarrow \mathbb{R}_{+}$and $V: L \rightarrow \mathbb{R}_{+}$that satisfy the following conditions.

(1) Advisers' optimality:

$$
V(l)=\max _{\tilde{b} \in B \cup\{\emptyset\}} v(\tilde{b}, l, U(\tilde{b}))
$$

and $(b, l) \in \operatorname{supp} H$ if and only if $b$ maximizes (5) given $l$. 
(2) Customers' optimality:

$$
U(b)=\max _{\tilde{l} \in L \cup\{\emptyset\}} u(b, \tilde{l}, V(\tilde{l}))
$$

and $(b, l) \in \operatorname{supp} H$ if and only if $l$ maximizes (6) given $b$.

(3) Feasibility Condition in Equation (4).

The definition does not explicitly define the information provided in equilibrium. However, given equilibrium payoffs $V(l)$ and $U(b)$, it is implicitly defined via Problem (3) and it is characterized in details below.

\section{Characterization}

We solve the model in two steps. First, we show that within a pair, without loss of generality, the optimal policy in Problem 3 can be characterized by a cutoff $\hat{s} \in[b \lambda, \lambda]$ such that the adviser recommends to invest if and only if $s \geq \hat{s}$ (Proposition 1). As a result, despite allowing for a general message space, we obtain a simple functional form for $v(b, l, u)$. Based on that, we then analyze the sorting decision in this two-sided market, and pin down the equilibrium payoff and information policies for all agents.

\subsection{Information Policies}

Our information structure implies that whenever a customer receives a bad signal $(x=0)$, he knows for sure that the asset is bad (i.e., $x=0$ whenever $s<b \lambda$ which implies that $y(s, l)<0$ with certainty). Then, investing is never optimal for any message received from an adviser. We we can therefore limit attention to $x=1$ when describing the optimal information policy. Conditional on $x=1$, the customer learns that $s \geq b \lambda$ and her belief about $s$ is given by the pdf $\frac{f(s)}{1-F(b \lambda)}$ with domain $[b \lambda, 1]$. We write $\mu_{1}$ for the corresponding measure.

Reformulation Given any policy $\sigma$ and realized message $m$, a customer forms a posterior belief $\mu \in \Delta([0,1])$. Since the message is random, $\sigma$ induces a distribution $P_{\sigma}$ on the space of posteriors beliefs. Based on Kamenica and Gentzkow (2011), Prop. 1, every distribution 
on the space of posterior beliefs $P \in \Delta(\Delta([b \lambda, 1]))$ can be induced by a signal, provided that $P$ satisfies the Bayes-plausibility condition

$$
E_{P} \mu=\mu_{1}
$$

We can therefore represent the adviser's problem 3 as maximizing over Bayes plausible distributions of posteriors after $x=1$ has realized and identify any information policy with $P$.

We let $i(\mu) \in\{0,1\}$ denote the investment decision conditional on $x=1$ and posterior $\mu$, so that $i=1$ whenever the customer's expected value from investing is positive and $i=0$ otherwise, i.e.

$$
i(\mu)=\mathbb{1}\left\{E_{\mu}[y(s, l)]-\alpha_{c} \geq 0\right\} .
$$

Given given information policy $P$, the probability of a customer investing is then given by $E_{P}[i(\mu)]$. Hence, the payoff of an adviser under such policy is

$$
\tilde{V}(b, l, P)=\alpha(1-F(b \lambda)) E_{P}[i(\mu)] .
$$

That is, conditional on the customer receiving a positive private signal, which happens with probability $1-F(b \lambda)$, the adviser receives $\alpha$ with probability $E_{P}[i(\mu)]$. The expected utility of a customer under information policy $P$ is then his expected payoff conditional on investing, which is

$$
\tilde{U}(b, l, P)=(1-F(b \lambda)) E_{P}\left[i(\mu)\left(E_{\mu}[y(s, l)]-\alpha_{c}\right)\right] .
$$

To summarize, the optimization problem of an adviser in Equation 3 can be written as

$$
\begin{aligned}
v(b, l, \bar{u})= & \max _{P \in \Delta(\Delta([b \lambda, 1]))} \alpha(1-F(b \lambda)) E_{P}[i(\mu)] \\
\text { s.t. } & (1-F(b \lambda)) E_{P}\left[i(\mu)\left(E_{\mu}[y(s, l)]-\alpha_{c}\right)\right]-u^{0}(b, l) \geq \bar{u} \\
& E_{P} \mu=\mu_{1} .
\end{aligned}
$$

Threshold Policy We now solve for the optimal policy and show that it is a threshold policy. The following Lemma shows that the optimal policy induces at most two posterior beliefs. 
Lemma 1. For any information policy $P$ such that $\tilde{U}(b, l, P)>u^{0}(b, l)$, there exists an equivalent policy $\hat{P}$, which only puts weight

$$
p:=P(\mu: i(\mu)=1)
$$

on the posterior $\mu_{I}=E_{P}[\mu \mid i(\mu)=1]$ and $(1-p)$ on the posterior $\mu_{N}=E_{P}[\mu \mid i(\mu)=0] .^{11}$

Based on Lemma 1, it is without loss of generality to put mass on two posteriors $\mu_{I}$ and $\mu_{N}$, which are the posteriors conditional on investing and not investing. The Bayes plausibility condition then becomes

$$
p \mu_{I}+(1-p) \mu_{N}=\mu_{1}
$$

It immediately implies that $\mu_{I}$ and $\mu_{N}$ are absolutely continuous with respect to $\mu_{1}$. Therefore, they both must admit densities, which, with slight abuse of notation, we denote with $\mu_{I}(s)$ and $\mu_{N}(s)$. For any given state $s$, the density $\mu_{N}(s)$ can be expressed as a function of $p$ and $\mu_{I}(s)$ using the Bayes plausibility condition. This implies

$$
\mu_{N}(s)=\frac{\frac{f(s)}{1-F(b \lambda)}-p \mu_{I}(s)}{1-p} \geq 0
$$

which is strictly decreasing in $\mu_{I}(s)$. Since the density function must be positive, this equation defines an upper bound for $\mu_{I}(s)$.

Hence, the adviser's problem can be further reduced to maximize over $\mu_{I}(s)$ and $p$, subject to

$$
\mu_{I}(s) \in\left[0, \frac{1}{p} \frac{f(s)}{1-F(b \lambda)}\right] .
$$

Recall that an adviser only cares about the probability of investing (which is represented by $p$ ). However, for a given $p$ and conditional on the investment taking place, a customer is strictly better off if an adviser puts a higher weight on the states that give a positive payoff, i.e. $s \geq \lambda$. This immediately suggests that for any optimal policy that solves Problem 3 ,

\footnotetext{
${ }^{11}$ In equilibrium, all customers who participate in the market will receive a value strictly higher than $u^{0}(b, l)$. Throughout this Section, we therefore focus attention on the case $\tilde{U}(b, l, P)>u^{0}(b, l)$. If $\tilde{U}(b, l, P)=u^{0}(b, l)$, the customer receives no information. $P$ then simply puts probability one on the customer's prior.
} 
$\mu_{I}(s)$ must hit its upper bound for any $s \geq \lambda$. Otherwise, there exists a Pareto improvement. On the other hand, whenever $s<\lambda$, customers receive a constant payoff of $-l$. Hence, on $[0, \lambda]$ it is irrelevant for customers whether $\mu_{I}(s)$ hits is upper bound. Only the total mass on $s<\lambda$ matters.

As a result, without loss of generality, the optimal policy can be characterized by a cutoff $\hat{s} \in[b \lambda, \lambda]$ such that, for any $s \geq \hat{s}$, the density $\mu_{I}(s)$ hits its upper bound and is zero otherwise:

$$
\mu_{I}(s)=\left\{\begin{array}{cc}
\frac{1}{p} \frac{f(s)}{1-F(b \lambda)} & s \geq \hat{s} \\
0 & s<\hat{s} .
\end{array}\right.
$$

The probability of investing is then given by $p=1-F(\hat{s})$. This leads to our Proposition 1 .

Proposition 1. Without loss of generality, the optimal policy can be characterized by a cutoff $\hat{s} \in[b \lambda, \lambda]$ such that the adviser recommends to invest if and only if $s \geq \hat{s}$.

The implementation of this policy is a message $m(s)=\mathbb{1}\{s \geq \hat{s}\}$. That is, when the adviser recommends to (not) invest, the state $s$ must be (lower) higher than a cutoff $\hat{s} \in$ $[b \lambda, \lambda]$. In the following, we identify each information policy with the cutoff $\hat{s}$.

The higher the threshold $\hat{s}$, the better the information, as the customer is less likely to have a false positive. The gain of a customer who seeks advice about asset $l$ with information policy $\hat{s}$, relativity to the self-directed trade, thus yields

$$
\tilde{U}(b, l, \hat{s})-u^{0}(b, l)=(F(\hat{s})-F(b \lambda)) l-\alpha_{c}(1-F(\hat{s})) .
$$

The first term captures the additional gain from information $\hat{s}$. Specifically, under the cutoff rule $\hat{s} \in[b \lambda, \lambda]$, a customer $b$ makes a bad investment and receives a negative payoff $-l$ with probability $F(\lambda)-F(\hat{s})$. If a customer would have traded based on his own signal, he would have made a mistake with probability $F(\lambda)-F(b \lambda)$. Hence, the added value of cutoff rule $\hat{s}$ to a customer $b$ is given by $(F(\hat{s})-F(b \lambda)) l$, which represents the value of reducing the customer's mistakes. The second term represents the expected cost by investing through an adviser. That is, the customer needs to pay additional cost $\alpha_{c}>0$ whenever he invests, which happens with probability $1-F(\hat{s})$. 
The adviser's optimization problem can now be conveniently rewritten as

$$
\begin{aligned}
& v(b, l, \bar{u}) \equiv \max _{\hat{s} \in[b \lambda, \lambda]} \alpha(1-F(\hat{s})) \\
& \text { s.t. }(F(\hat{s})-F(b \lambda)) l-\alpha_{c}(1-F(\hat{s})) \geq \bar{u} .
\end{aligned}
$$

An adviser is better off for a lower $\hat{s}$, as it implies a higher probability of investment. A customer, on the other hand, is worse off for a lower $\hat{s}$, as it implies a higher probability of making mistakes.

The solution to Problem 3 admits a simple closed form:

$$
v(b, l, \bar{u})=\alpha\left(1-\left(\frac{F(b \lambda) l+\bar{u}+\alpha_{c}}{l+\alpha_{c}}\right)\right) .
$$

Similarly, we write $u(b, l, \bar{v})$ for the gain of customer $b$ who matches with adviser $l$, who receives utility $\bar{v}$. The expression is given by

$$
u(b, l, \bar{v})=(1-F(b \lambda)) l-\left(\frac{l+\alpha_{c}}{\alpha}\right) \bar{v} .
$$

\subsection{Matching Patterns}

We now use the solution obtained in Equation (12) to analyze the matching patterns. First of all, according to Equation (12), conditional on providing $\bar{u}$ to her customer, an adviser would prefer to match with less informed customer, because $v_{b}(b, l, \bar{u})<0$. Intuitively, fixing any policy $\hat{s}$, a less informed customer gains more as he has worse information when he trades the asset himself. Hence, conditional on promising gain $\bar{u}$ to a customer, an adviser is able to give worse information (i.e. a lower threshold $\hat{s}$ ) if the customer is less informed. Thus, she obtains a higher payoff.

In equilibrium, an adviser takes customers' utilities $U(b)$ as given and chooses a customer optimally:

$$
V(l)=\max _{\tilde{b}} v(\tilde{b}, l, U(\tilde{b})) .
$$

Clearly, the payoff to an adviser decreases with the promised utility of the customer, 
since $v_{u}(b, l, \bar{u})<0$. Given that all advisers prefer to match with less informed customers, competition implies that less informed customers must be promised with a higher utility $U(b)$ in equilibrium. Otherwise, everyone wants to choose a lower $b$, which cannot happen in equilibrium. This thus suggests that the equilibrium gain $U(b)$ must decrease in $b$. Similarly, an adviser with more valuable expertise is the more attractive type from the viewpoint of customers (i.e., $u_{l}(b, l, \bar{v})>0$ ) and thus the equilibrium profit $V(l)$ must increase in $l$.

In equilibrium, it is the adviser that can offer the most valuable information that attracts the least informed customer. To understand the sorting outcome, observe that, fixing a policy $\hat{s}$ for any pair $(l, b)$, the information gain of customer is given by $(F(\hat{s})-F(b \lambda)) l$. That is, there is complementarity between a higher $l$ and a less informed customer for any given information. This suggests that it is less costly for an adviser with higher $l$ to attract the least informed customer, since he can lie more (i,.e., providing a lower $\hat{s}$ ) conditional on providing the same level of promised utility.

One can see this from customers' viewpoint as well. Since the gain of any given policy is highest for least informed customers, they are the ones who are most willing to accept a less precise policy in exchange for more valuable expertise. Formally, from Equation (13), one can easily see that it is the less informed customer that prefer to seek for advice on higher $l$ in equilibrium. For any $l^{\prime}>l$ and $b^{\prime}>b$,

$$
\begin{aligned}
& u\left(b, l^{\prime}, V\left(l^{\prime}\right)\right)-u\left(b^{\prime}, l^{\prime}, V\left(l^{\prime}\right)\right) \\
= & \left(F\left(b^{\prime} \lambda\right)-F(b \lambda)\right) l^{\prime} \\
> & u(b, l, V(l))-u\left(b^{\prime}, l, V(l)\right)
\end{aligned}
$$

Hence, $u\left(b^{\prime}, l^{\prime}, V\left(l^{\prime}\right)\right)-u(b, l, V(l)) \geq 0$, then $u\left(b, l^{\prime}, V\left(l^{\prime}\right)\right)>u(b, l, U(b))$. That is, if a more informed customer $b^{\prime}$ weakly prefer an adviser with higher $l^{\prime}$, then a less informed customer must strictly prefer such an advise. ${ }^{12}$ As a result, the equilibrium must display negative associative matching.

Lemma 2 (Assortative Matching). A less informed customer (a lower b) matches to an adviser with more valuable expertise (a higher $l$ ).

\footnotetext{
${ }^{12}$ One can establish the single crossing property for $v(b, l, U(b))$ as well. That is, if an adviser with a lower $l$ prefers a less informed customer, then an adviser with a higher $l$ must strictly prefer such a customer. See Appendix.
} 


\subsection{Equilibrium Disclosure and Payoff}

We now solve for the assignment function and the information received by each customer directly. These two functions can then easily be mapped to the and equilibrium payoffs $U(b)$ and $V(l)$.

Let $\ell: B \rightarrow L \cup\{\emptyset\}$ denote the type of adviser $l$ from whom customer $b$ receives information. Lemma 2 suggests that the assignment function $\ell(b)$ must be weakly decreasing. Moreover, we know that (1) less informed customers must gain more by participating in the market (i.e. $U(b)$ decreases in $b$ ) and (2) advisers with more valuable expertise must obtain a higher equilibrium payoff (i.e., $V(l)$ increases in $l$ ). This can be seen from Equation (14), where by the envelope theorem $V^{\prime}(l) \geq 0$.

Hence, in equilibrium there exists a highest active customer (denoted by $b^{*}$ ) and a lowest active adviser (denoted by $l^{*}$ ) such that $Q\left(b^{*}\right)=1-G\left(l^{*}\right)$.

For agents that are actively matched, the assignment function must then solve the following market-clearing condition:

$$
\int_{\underline{b}}^{b} d Q(\tilde{b})=\int_{\ell(b)}^{\bar{l}} d G(\tilde{l})
$$

That is, given any $b$, the measure of customers below $b$ (LHS) equals the measure of advisers above $\ell(b)$ (RHS). This yields the differential equation

$$
\frac{d \ell(b)}{d b}=-\frac{d Q(b)}{d G(\ell(b))} \leq 0,
$$

with initial condition $\ell(\underline{b})=\bar{l}$. That is, the least informed customer must match with the adviser with the highest $l$.

Let $s^{*}(b)$ denote the equilibrium information policy that an active customer $b$ receives. The gain for this customer $U(b)$ depends on $\ell(b)$, the asset about which he receives advice, and the information policy $s^{*}(b)$. We have

$$
U(b)=\left(F\left(s^{*}(b)\right)-F(b \lambda)\right) \ell(b)-\alpha_{c}\left(1-F\left(s^{*}(b)\right)\right) .
$$


In equilibrium, the FOC of the advisers' optimization problem in (14) must satisfy

$$
U^{\prime}(b)=-F^{\prime}(b \lambda) \lambda \ell(b)<0
$$

so that it is indeed optimal for an adviser $\ell(b)$ to match with customer $b$. Taking derivatives with respect to $b$ in Equation (17), together with Equation (18) yields the following differential equation for $s^{*}(b)$ :

$$
\frac{d s^{*}(b)}{d b}=-\frac{\left(F\left(s^{*}(b)\right)-F(b \lambda)\right) \ell^{\prime}(b)}{F^{\prime}\left(s^{*}(b)\right)\left(\ell(b)+\alpha_{c}\right)} \geq 0
$$

That is, a more informed customer must receive more precise information (i.e., a higher cutoff $\hat{s}$ ) in equilibrium, despite having lower gain $U(b)$. On the other hand, an adviser with more information sensitive asset matches with a less informed customer. Thus she provides worse information (i.e. a lower cutoff) and earns a higher profit. Formally, each adviser's payoff is given by

$$
V(l)=\alpha\left(1-F\left(s^{*}\left(\ell^{-1}(l)\right)\right)\right.
$$

where $\ell^{-1}(l)$ denotes the inverse of $\ell$.

Any information policy must satisfy customers' participation constraint in equilibrium. To facilitate our characterization, we define $s^{0}(b, l)$ as the policy under which the participation constraint of a customer with type $b$ binds. ${ }^{13}$ That is,

$$
s^{0}(b, l) \equiv \min \left\{\hat{s} \in[b \lambda, \lambda]: \tilde{U}(b, l, \hat{s})-u^{0}(b, l) \geq 0\right\}
$$

The IR constraint then simply requires that $s^{*}(b) \geq s^{0}(b, \ell(b)) \forall b$. The equilibrium characterization is summarized in Proposition 2.

Proposition 2. The equilibrium is characterized by a marginal customer $b^{*}$, an assignment function $\ell(b): B \rightarrow L \cup\{\emptyset\}$, and an information policy $s^{*}(b):\left[0, b^{*}\right] \rightarrow[0, \lambda]$ such that

(1) The marginal customer is given by $b^{*}=\left\{\begin{array}{cl}Q^{-1}(1) & \text { if } Q(\bar{b})>1 \\ \bar{b} & \text { if } Q(\bar{b}) \leq 1\end{array}\right.$

\footnotetext{
${ }^{13}$ Our assumption $(A 2)$ guarantees the solution $s^{0}(b, l) \in[b \lambda, \lambda]$ exists. To see this, let $z(\hat{s}, l, b) \equiv$ $(F(\hat{s})-F(b \lambda)) l-\alpha_{c}(1-F(\hat{s}))=0 \forall b, \forall l$. Given that $z_{s}=\left(l+\alpha_{c}\right)>0$, Assumption (A2) implies that $z(\lambda, \underline{l}, \bar{b})>0$ and that there exists $\hat{s} \in[b \lambda, \lambda]$ such that $z(\hat{s}, \underline{l}, \bar{b})=0$. Furthermore, given that $z_{b}<0$, and $z_{l}>0, s^{0}(b, l)<s^{0}(\bar{b}, \underline{l})<\lambda \forall b, l$.
} 
(2) For any $b \leq b^{*}, \ell(b)$, and $s^{*}(b)$ solve the system of differential equations (16) and (19) with initial conditions $\ell(\underline{b})=\bar{l}$ and

$$
s^{*}\left(b^{*}\right)=\left\{\begin{array}{cc}
s^{0}\left(b^{*}, \ell\left(b^{*}\right)\right) & \text { if } Q(\bar{b})>1 \\
\lambda & \text { if } Q(\bar{b})<1 \\
\zeta & \text { if } Q(\bar{b})=1
\end{array}\right.
$$

where $\zeta \in\left[s^{0}\left(b^{*}, \ell\left(b^{*}\right)\right), \lambda\right]$. For $b>b^{*}$, customers remain inactive, i.e. $\ell(b)=\emptyset$.

Note that the initial condition pinning down $s^{*}\left(b^{*}\right)$, which represents the information received by the marginal customer, crucially depends on whether customers or advisers are on the short side of market. If advisers are on the short side of the market (i.e., $Q(\bar{b})>1)$, the marginal customer $b^{*}$ is the one for which $Q\left(b^{*}\right)=1$. This customer will gain zero by participating in the market, i.e. $U\left(b^{*}\right)=0$, and therefore he receives his autarky value and $s^{0}\left(b^{*}, \ell\left(b^{*}\right)\right)$.

On the other hand, if customers are on the short side of the market, i.e. $Q(\bar{b})<1$, the marginal adviser $l^{*}$ is given by $Q(\bar{b})=1-G\left(l^{*}\right)$. Since customers are scarce in this case, the marginal adviser must provide her customer with as much as information as possible (i.e, full disclosure, so that $s^{*}\left(b^{*}\right)=\lambda$ ). Otherwise, other inactive advisers can outbid her by providing better information to attract her customer. Lastly, when $Q(\bar{b})=1$, customers' share is not uniquely pin down. So, any information between $s^{0}\left(b^{*}, \ell\left(b^{*}\right)\right)$ and $\lambda$ can be supported as an equilibrium. This explains condition (2) in the Proposition.

In summary, as information is the only currency, each adviser attracts customers by promising a certain information value. In equilibrium, each adviser must prefer attracting her customer at his equilibrium pay level (in terms of information) to attracting any other customers at their pay level.

Similarly, given any information provided by an adviser, it is optimal for customers to choose their optimal adviser. In particular, since an adviser with higher $l$ provides less valuable information, a customer is effectively facing a trade-off between choosing more informative information (a lower $\hat{s}$ ) v.s. more valuable knowledge (a higher $l$ ). Defining $s^{*}(l) \equiv s^{*}\left(\ell^{-1}(l)\right)$, we can rewrite a customer's choice as

$$
U(b)=\max _{l}\left(F\left(s^{*}(l)\right)-F(b \lambda)\right) l-\alpha_{c}\left(1-F\left(s^{*}(l)\right)\right) .
$$



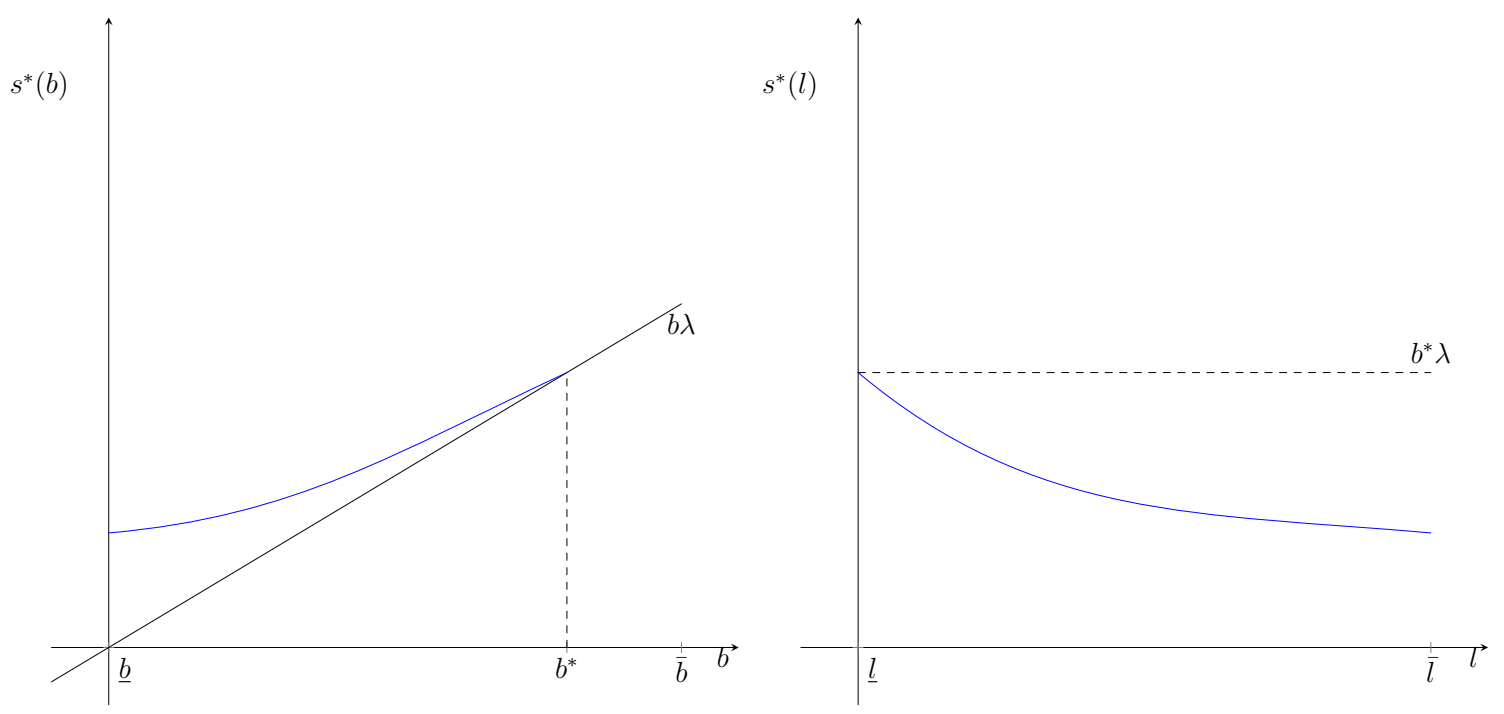

Figure 3: Equilibrium Information Policy with Scarce Advisers $\left(\alpha_{0}>0, \alpha_{c}=0\right)$

That is, the equilibrium information schedule $s^{*}(l)$ is such that all customers prefer to match to his adviser $\ell(b)$. In equilibrium, a less informed customer chooses a higher $l$ with less precise information (i.e., a lower $s^{*}(l)$ ).

The equilibrium information policy is illustrated in Figure 3.

\section{Comparative Statics}

Composition Effects One unique feature of our market with two-sided heterogeneity is that competition is about which customer to serve and which adviser to ask for advice. In equilibrium, all agents are compensated so that it is indeed optimal for them to match to their counterparty instead of others. Hence, the distribution of other customers and advisers, which represents the potential competitors, is the key determinant of information provision. In fact, if if all advisers were the same, the equilibrium will always feature one information policy and the composition of distribution does not matter. ${ }^{14}$

To further explore the effects of heterogeneity, we now consider comparative statics on

\footnotetext{
${ }^{14}$ Similar to the logic that there exists one price that clears the market, there will be one information policy that makes sure the marginal customer is willing to participate. That is, if advisers (customers) are at the short side, $s^{*}(b)=b^{*} \lambda\left(s^{*}(b)=\lambda\right)$.
} 
the distribution of customer types and analyze how the composition affects the quality of information in equilibrium. We consider a change in the shape of distribution $Q(b)$ within the active matches. That is, fixing the marginal type $b^{*}$. Formally, consider two distributions $Q_{1}(b)$ and $Q_{2}(b)$ : both of them have the same range $B$ but $Q_{1}(b)$ first-order stochastically dominates $Q_{2}(b)$, i.e. $Q_{1}(b) \leq Q_{2}(b) \forall b \in B$. Intuitively, $Q_{2}(b)$ has more customers who are relatively uninformed.

To see the effect on information provision more clearly, it is convenient to look at the information policy as a function of adviser $l$. The differential equation for information policies in (19) can be rewritten as

$$
\frac{d s^{*}(l)}{d l}=-\frac{F\left(s^{*}(l)\right)-F\left(\ell^{-1}(l) \lambda\right)}{F^{\prime}\left(s^{*}(l)\right)\left(l+\alpha_{c}\right)},
$$

where the initial condition for the marginal adviser $l^{*}$ is given by $s^{0}\left(b^{*}, \ell\left(b^{*}\right)\right)$, which is the same for given fixed $b^{*}$.

When there are more customers who are less informed, any given adviser $l$ must match with a customer with a (weakly) lower $b$. Thus, $\ell_{2}^{-1}(l) \leq \ell_{1}^{-1}(l)$, where $\ell_{j}^{-1}(l)$ represents the assignment under distribution $Q_{j}(b)$. Hence, one can show via a comparison theorem, that the solution to Equation (22) under $Q_{2}(b)$ must be weakly lower. That is, when the economy has a larger portion of less informed customers, information quality is worse for all customers, not just the ones who are less informed. This is in contrast to settings with either a monopoly or with homogeneous advisers. In those environments, the aggregate distribution will not affect the information provided by an adviser $l$ so there is no composition effect.

This result highlights how competitive forces shape information provision. Advisers compete for less informed customers, since they are the ones who value information most, and are also easier to deceive. As a result, these customers earn rents. Now, fixing a customer $\hat{b}$, when all other customers above him becomes more informed, meaning that his other competitors becomes less attractive, such a customer then must earn a higher surplus, thus receiving better information.

One can also consider a similar exercise by changing the shape of the distribution of adviser types $G(l)$. For example, suppose $G_{1}(l)$ first-order stochastically dominates $G_{2}(l)$. That is, there are less advisers with valuable information under $G_{2}(l)$. Same intuition as before applies: an adviser with expertise in higher $l$ becomes more scarce and as a result, 
she must earn a higher profit, meaning worse information for customers. The comparative statics are summarized in Lemma 3.

Lemma 3. (1) Consider two distributions with the same range and $Q_{1}(b) \leq Q_{2}(b)$, then $s^{*}(l)$ is weakly lower under $Q_{2}(b)$. (2) Consider two distributions with the same range and $G_{1}(l) \leq G_{2}(l)$, then $s^{*}(l)$ is weakly lower under $G_{2}(l)$.

Advisers Fees Intuitively, one might expect that a higher fee worsens information, because it implies a more sever conflict of interest. This argument is inaccurate under a competitive market. From equation (21), we see that commission fee $\alpha_{c}$ and information are substitutes from the viewpoint of customers. Hence, a customer must be compensated with a better information whenever there is a higher commission fee $\alpha_{c}$. In particular, given any commission fees $\alpha_{c}>0$, the schedule of equilibrium information $s^{*}\left(l ; \alpha_{c}\right)$ is pinned down in a way so that all customers must choose their advisers $\ell(b)$. This suggests that the higher the commission fees, the better the information being provided in equilibrium.

Lemma 4. Let $s^{*}\left(l ; \alpha_{c}\right)$ denote the equilibrium information under commission $\alpha_{c}$. The higher the fee, the better the information: for any $\alpha_{c}^{\prime}>\alpha_{c}, s^{*}\left(l ; \alpha_{c}^{\prime}\right)>s^{*}\left(l ; \alpha_{c}\right)$.

Scaling Effects We now turn to another source of the conflict of interest: $\alpha_{0}$ which captures other potential gains of advisers that are not costly to customers. From Equation (22), we can immediately show that the information policy is independent of $\alpha_{0}$. In other words, a higher $\alpha_{0}$ only scales up advisers' profit. Similarly, when $\alpha_{c}=0$, if all assets $l$ are scaled by a factor $z>0$, (i.e., $l_{z}=z l$ ) the customer of each adviser remains the same. One can then show that the equilibrium information policy remains the same as well, i.e., $s_{z}^{*}\left(l_{z}\right)=s^{*}\left(\frac{l_{z}}{z}\right)$. In summary, when $\alpha_{0}$ increases (or when the loss of each asset increases), the payoff to all advisers (or to customers) simply increases by the same amount.

Lemma 5. For any $z>0$, (1) If $\alpha_{c}=0$ and $l_{z} \equiv z l$, the information policy stays the same. We have $s_{z}^{*}(b)=s^{*}(b), U_{z}(b)=z U(b)$, and $V_{z}\left(l_{z}\right)=V(l)$. (2) If $\alpha_{z} \equiv z \alpha$, the information policy stays the same and we have $s_{z}^{*}(b)=s^{*}(b), U_{z}(b)=U(b)$, and $V_{z}(l)=z V(l)$. 


\section{Information Distortion}

Our equilibrium definition guarantees that the equilibrium allocation is always on the Pareto frontier. However, it is well known that when utilities are not fully transferable, the equilibrium outcome of matching markets might not maximize total surplus. To see this explicitly in our model, consider a social planner who designs the allocation function and the policy function $s^{e}(b, l)$ to maximize the pair-wise surplus, taking into account the gains for agents on both sides of the market.

Within each pair, the information policy $s^{e}(b, l)$ thus solves

$$
\Omega^{e}(b, l)=\max _{s \in[b \lambda, \lambda]} \alpha_{0}(1-F(s))+(F(s)-F(b \lambda)) l .
$$

Since $\alpha_{c}$ only affects the transfer within the pair, it does not affect the the pair-wise surplus. The solution is then simply given by

$$
s^{e}(b, l)=\left\{\begin{array}{cl}
\lambda & \text { if } l \geq \alpha_{0} \\
b \lambda & \text { if } l<\alpha_{0} .
\end{array}\right.
$$

That is, when the benefit of the adviser $\alpha_{0}$ is strictly smaller than the loss $l$, the information policy that maximizes the joint surplus is full disclosure. If $\alpha_{0}$ is larger than $l$, no disclosure is optimal. Hence, the surplus function yields:

$$
\Omega^{e}(b, l)=\left\{\begin{array}{cc}
\alpha_{0}(1-F(\lambda))+(F(\lambda)-F(b \lambda)) l & \text { if } l \geq \alpha_{0} \\
\alpha_{0}(1-F(b \lambda)) & \text { if } l<\alpha_{0}
\end{array}\right.
$$

For either case, the surplus function is sub-modular, i.e. $\Omega_{b l}^{e} \leq 0$, so the maximal surplus can be achieved by negative sorting. Thus, the assignment function is the same under the decentralized equilibrium and the planner problem.

On the other hand, the information policy generally is distorted in the decentralized equilibrium, as the information policy generally lies in between full disclosure and no disclosure. In other words, the decentralized equilibrium can feature either too little information (when $\alpha_{0}<l$ ) or too much (when $\alpha_{0}>l$ ).

Given that the sorting pattern in the decentralized market will be the same as the solution 
to the social planner problem (i.e., the same the assignment function $\ell(b)$ and same marginal customer $b^{*}$ ), the welfare loss is given by the distortion on the information policy relative to the planner's solution, which yields

$$
L=\int_{\underline{b}}^{b^{*}}\left|s^{e}(b, \ell(b))-s^{*}(b)\right| \ell(b) d Q(b) .
$$

In the case where full disclosure is optimal, we have $s^{e}(b, \ell(b))=\lambda \forall b$. Hence, combining this with our results in Lemmas 3 and 4, information distortion increases when there are more uninformed customers, less advisers with valuable expertise, and, perhaps counter-intuitively, a lower commission fee.

\section{Application: Market for Financial Advisers}

We now apply our model to the market for financial advisers, where conflicts of interest arise when advisers receive so-called conflicted payments. In these markets, payments received by advisers are typically borne by customers. Hence, we now interpret $\alpha_{c}>0$ as the conflicted payment and set $\alpha_{0}=0$ for simplicity. Specifically, in the case of mutual funds, for example, investors can either purchase a broker-channel mutual fund by paying additional distribution fees $\alpha$ c (e.g., loads and 12b-1 fees) or buy direct-channel funds without distribution fees. ${ }^{15}$

We now use our result to shed light on how fee structures affect information provision and customer welfare. To further highlight the costs generated by these misaligned incentives, we compare our result to an environment where advisers can set competitive fees that are non-conflicted payments. As discussed in a White House report (CEA (2015)), advisers can receive two types of compensation: (1) conflicted payments and (2) payments that do not directly depend on customers' investment decisions, such as hourly rates. Thus, we further extend our model to allow for non-conflicted, fixed payments to advisers whenever a customer asks for advice (denoted by $\gamma \geq 0$ ).

Thus, any difference between our main model and the benchmark with competitive fees represents the cost of a compensation structure having conflicts of interests. Perhaps sur-

\footnotetext{
${ }^{15}$ For example, Bergstresser et al. (2009) estimates that broker-channel mutual fund consumers may have paid as much as $\$ 3.6$ billion in front end loads in $2002, \$ 2.8$ billion in back-end loads and another $\$ 8.8$ billion in $12 \mathrm{~b}-1$ fees, in additional to the investment management fees.
} 
prisingly, we show that despite information being distorted in our main model, customer welfare is not harmed as long as advisers are on the short side of the market. Our result thus invalidates the conventional argument that banning conflicted payment improves customer welfare.

\subsection{Benchmark with Competitive Fees}

In an environment where fees can be set by advisers, the fees will be determined in equilibrium together with the information policy. Suppose an adviser can post fees $\left(\alpha_{c}, \gamma\right)$ in addition to the information policy $\sigma$. As before, customers make their matching decisions to maximize their expected utility of the match. This setting can then be solved as the classical assignment model with transferable utilities, where the optimal information policy simply maximizes the pairwise surplus. ${ }^{16}$ Since we are in the case that $\alpha_{0}=0$, the optimal policy $s^{e}(b, l)$ is full disclosure as shown in equation (24).

Hence, the pairwise surplus is given by $\Omega^{e}(b, l)=(F(\lambda)-F(b \lambda)) l$. Note that, since $\Omega_{b l}<0$, the equilibrium still features negative sorting. The utility of a customer is

$$
U^{F B}(b) \equiv \max _{l} \Omega^{e}(b, l)-V(l),
$$

where, by the envelope theorem,

$$
\frac{d U^{F B}(b)}{d b}=-F^{\prime}(b \lambda) \lambda \ell(b) .
$$

Equation (25) shows that for any given assignment function $\ell(b)$, the utility of a customer $U(b)$ is pinned down up to a constant. Specifically, given the utility level of the marginal customer $U^{F B}\left(b^{*}\right)$, customers' utilities and thus total payments are pinned down uniquely. Clearly, what matters is the total payment but not the composition of the fees.

The level of the marginal customer is pinned down in the standard way: when advisers are scarce, the marginal customer must gain zero after the fee. That is, he pays $(F(\lambda)-$ $F(b \lambda)) \ell\left(b^{*}\right)$ for full disclosure. On the other hand, when customers are scarce, the marginal customer receives the whole surplus. That is, he gets full disclosure for free.

\footnotetext{
${ }^{16}$ For example, Terviö (2008).
} 
Lemma 6. With competitive fees, all advisers provide full disclosure, and the gain of customers in equilibrium (after fees) is characterized by equation (25) with initial condition:

$$
U^{F B}\left(b^{*}\right)=\left\{\begin{array}{cc}
0 & \text { if } Q(\bar{b})>1 \\
(F(\lambda)-F(b \lambda)) \ell\left(b^{*}\right) & \text { if } Q(\bar{b})<1 \\
U^{0}(\bar{b}) & \text { if } Q(\bar{b})=1
\end{array}\right.
$$

where $b^{*}=\min \left\{Q^{-1}(1), \bar{b}\right\}, 1-G\left(\ell\left(b^{*}\right)\right)=Q\left(b^{*}\right)$, and $U^{0}(\bar{b}) \in[0,(F(\lambda)-F(\bar{b} \lambda)) \underline{l}]$.

\subsection{Equivalence of Competition in Information vs. Fees}

When fees cannot be freely adjusted, as shown in our baseline model, advisers compete on another dimension: information. We now use our results with conflicted fees $\alpha_{c}>0$ to answer two questions: (1) What is the cost to customers under conflicted payments? (2) How does the fee affect information quality?

Equivalence of Customers' Utilities Recall that the utility of a customer in equilibrium is characterized by Equation (18), $U^{\prime}(b)=F^{\prime}(b \lambda) \lambda \ell(b)$, which is in fact identical to our competitive benchmark in equation (25). As a result, the difference (if any) across these two environments is captured by the utility level of the marginal customer, which is summarized by Proposition 3:

Proposition 3. For any conflicted payment $\alpha_{c}$ that satisfies A1 and A2, the utility of each customer is given by

$$
U(b)=\left\{\begin{array}{cl}
U^{F B}(b) & \text { if } Q(\bar{b}) \geq 1 \\
U^{F B}(b)-\alpha_{c}(1-F(\lambda)) & \text { if } Q(\bar{b})>1
\end{array}\right.
$$

where $U^{F B}(b)$ denotes customer utilities under the competitive benchmark.

The proposition establishes a connection between competition via fees and competition via information provision. To see this more clearly, consider any given commission fee $\alpha_{c}$ 
and information policy $s^{*}(l)$. The equilibrium gain of customer $b$ can rewritten as:

$$
U(b)=\max _{l}-F(b \lambda) l+\tau(l)
$$

where $\tau(l) \equiv F\left(s^{*}(l)\right) l-\alpha_{c}\left(1-F\left(s^{*}(l)\right)\right)$. That is, $\tau(l)$ can be interpreted as the net benefit provided by adviser $l$, which includes the value of information minus the fees.

In other words, the net benefit can take the form of either an informational or monetary reward. Nevertheless, any schedule $\tau(l)$ that guarantees that all customers optimally match to their own advisor $\ell(b)$ must satisfy the following FOC of customers' optimization:

$$
-F^{\prime}(b \lambda) \lambda \ell(b)=\tau^{\prime}(\ell(b))
$$

Hence, the schedule $\tau(l)$ is is uniquely pinned down up to a constant. ${ }^{17}$ In fact, this argument holds more generally for any exogenous fee structure that induces the same assignment function $\ell(b) .{ }^{18}$ In this case, consumer utilities can only be different up to a constant compared to the competitive benchmark, which is captured by the difference of the utility of the marginal customer $U\left(b^{*}\right)$.

The utility of the marginal customer is pinned down as usual: when the customers are in the short side of the market, the adviser of the marginal customer $\ell\left(b^{*}\right)$ must provide him the highest payoff possible. In this case, it means full disclosure minus the exogenous fees $\alpha_{c}(1-F(\lambda))$. Since a marginal customer would have received full disclosure for free under the benchmark, this implies that the net benefit of all customers will be lower by exactly the same amount, $\alpha_{c}(1-F(\lambda))$.

On the other hand, if advisers are on the short side, the marginal customer always receives zero gain. This is true as long as the fees are small enough so that the marginal customer can still gain by entering the market (i.e.,under A1 and A2). Hence, all customers must obtain the same net benefit $\tau(\ell(b))$ across the two settings: full disclosure but a higher fee or distorted information with a lower payment. In other words, how fees are set is in fact irrelevant for customers' utilities.

\footnotetext{
${ }^{17}$ This argument is analogous to revenue equivalence result in the standard pricing problem where customers have quasi-linear utility functions.

${ }^{18}$ In Appendix, we further allow for fees depending on types and provide condition under which $\left(\alpha_{c}(l), \gamma(l)\right)$ leads to negative sorting in equilibrium.
} 
Corollary 1 (Irrelevance). If advisers are on the short side, the net benefit received by all customers-a combination of fees and information-is the same under any fee structure that induces the same assignment function.

On the other hand, since the total surplus is strictly higher under the competitive benchmark (i.e., full disclosure), the profit of advisers must be higher, because customers' utilities are weakly lower. In other words, advisers are the one who bear the cost of information distortions under fixed fees. Combining with Lemma 4, the higher fee leads to a lower information distortion, thus a higher joint surplus and higher profit to advisers.

Corollary 2. If advisers are on the short side, the information distortion decreases the profit of advisers, but not of consumers. A higher fee $\alpha_{c}$ leads to a higher profits of all advisers.

\subsection{Empirical Implications}

One can compare returns across two different types of advisers: ones with conflicted payment and ones without. For those who are compensated by commission, the realized returns for investing asset $l$, taking into account the adviser's recommendation and commission fees, is then given by:

$$
R(l)=\frac{(1-F(\lambda)) r-\left(F(\lambda)-F\left(s^{*}(l)\right)\right) l}{\left(1-F\left(s^{*}(l)\right)\right)}-\alpha_{c} .
$$

One the other hand, without conflicted payments, the realized return will simply be $r$ given the full disclosure (i.e., $s^{*}(l)=\lambda \forall l$ ). However, they will need to pay a flat fee to adviser when asking for advice. Let $\gamma(l)$ denote the competitive fee charged by adviser $l$. Our theory predicts that customers will be in fact indifferent across these two types of fee structures, but simply pay in different ways. ${ }^{19}$ That is, the expected payoff of seeking advice is the same, which yields:

$$
\left(1-F\left(s^{*}(l)\right)\right) R(l)=(1-F(\lambda)) r-\gamma(l) .
$$

Empirically, the difference in these return is often interpreted as cost of conflicted payment, as discussed in CEA (2015). Our theory establishes that this interpretation is in fact misleading.

\footnotetext{
${ }^{19}$ The exercise here assumes that everything else is equal across these two types of structure. In particular, both $G(l)$ and $Q(b)$ are the same.
} 
The difference across these two only reflects the information quality, but customers can simply pay a higher fee for better information. Moreover, our theory also shows that the realized returns "before" fees, $R(l)+\alpha_{c}$, is the more precise measure of information, but not "after" fees.

Furthermore, the conditional returns do not measure the equilibrium value of advice, because they fail to subtract the (unobserved) outside option. Additionally, our result also highlights the importance of customers' selection effects and advisers' fixed effects. Without properly taking these into accounts, it appears that advice destroys value and customers are irrational. For example, if one naively compare performance among households who seek for advice vs. those who do not, one can easily conclude that those who trade through advisers have lower returns. However, according to our model, that is simply driven by the fact that customers who seek for advice are the less sophisticated ones.

\section{Conclusion}

The paper studies competitive market for information in which advisers are subject to conflicts of interests. In contrast to existing models that assume naïve customers, our model analyzes the quality of information in a competitive market with rational customers with different sophistication. We are thus able to establish new insights and predictions regarding customers' welfare and asset returns. Specifically, we show that it is the underlying distribution of financial literacy that determines the consumers' welfare. When advisers are scarce, the fee structure of advisers is irrelevant for the welfare of consumers. We further show that the existing empirical measures of conflicted advice can be misleading if it fails to take into account the selection effect of the characteristics of funds and customers. 


\section{A Proofs}

\section{A.1 Proof of Lemma 1}

The result follows from the law of iterated expectations. Consider an information policy $P$. The values of adviser and customer are

$$
\begin{aligned}
\tilde{V}(b, l, P) & =\alpha(1-F(b \lambda)) E_{P}[i(\mu)] \\
\tilde{U}(b, l, P) & =(1-F(b \lambda)) E_{P}\left[\max \left\{r \int_{\lambda}^{1} d \mu(s)-l \int_{b \lambda}^{\lambda} d \mu(s)-\alpha_{c}, 0\right\}\right] .
\end{aligned}
$$

Let $\mathcal{M}_{0}=\{\mu: i(\mu)=0\}$ denote the subset of the space of posterior beliefs where the customer does not invest and let $\mathcal{M}_{1}=\{\mu: i(\mu)=1\}$ denote the subset where he does. Since $U(p)>u^{0}(b, a)$, both $\mathcal{M}_{0}$ and $\mathcal{M}_{1}$ are non-empty. ${ }^{20}$ We have

$$
\tilde{V}(b, l, P)=\alpha(1-F(b \lambda)) p\left(\mathcal{M}_{1}\right)
$$

and

$$
\tilde{U}(b, l, P)=(1-F(b \lambda)) p\left(\mathcal{M}_{1}\right) E_{p}\left[r \int_{\lambda}^{1} d \mu(s)-l \int_{b \lambda}^{\lambda} d \mu(s)-\alpha_{c} \mid \mu \in \mathcal{M}_{1}\right]
$$

Now define $\mu_{N}=E_{p}\left[\mu \mid \mu \in \mathcal{M}_{0}\right], \mu_{I}=E_{p}\left[\mu \mid \mu \in \mathcal{M}_{1}\right]$, and $p=P\left(\mathcal{M}_{1}\right)$. Consider an information policy which puts mass $p$ on $\mu_{I}$ and mass $1-p$ on $\mu_{N}$. We have $i\left(\mu_{h}\right)=1$ and $i\left(\mu_{l}\right)=0$, and the ex-ante values under this alternative policy must be the same as under $P$ by the law of iterated expectations.

\section{A.2 Proof of Proposition 1}

The Bayes plausibility condition (7) is now

$$
p \mu_{I}+(1-p) \mu_{N}=\mu_{1}
$$

\footnotetext{
${ }^{20}$ If $\mathcal{M}_{1}$ were empty, then $U(p)=0$, which is below the customer's no-information value $u^{0}(b, a)$. If $\mathcal{M}_{0}$ were empty, then the customer always invests. But then necessarily $U(p)=u^{0}(b, a)$.
} 
which immediately implies that $\mu_{I}$ and $\mu_{N}$ are absolutely continuous with respect to $\mu_{1}$. Therefore, they both must admit densities, which we denote with $\mu_{I}(s)$ and $\mu_{N}(s)$. We denote the corresponding cdfs with $F_{I}$ and $F_{N}$.

The adviser's problem in (9) can therefore be written as

$$
\begin{aligned}
v(b, l, \bar{u})= & \max _{p, \mu_{I}, \mu_{N}} \alpha(1-F(b \lambda)) p \\
\text { s.t. } \quad & (1-F(b \lambda)) p\left(r\left(1-F_{I}(\lambda)\right)-l\left(F_{I}(\lambda)-F_{I}(b \lambda)\right)-\alpha_{c}\right)-u^{0}(b, l) \geq \bar{u} \\
& p \mu_{I}(s)+(1-p) \mu_{N}(s)=\frac{f(s)}{1-F(b \lambda)} \forall s \geq b \lambda \\
& \mu_{I}(s), \mu_{N}(s) \geq 0 \forall s \geq b \lambda \\
& \int_{b \lambda}^{1} \mu_{I}(s) d s=\int_{b \lambda}^{1} \mu_{N}(s) d s=1
\end{aligned}
$$

Using Bayes plausibility, we can solve for $\mu_{N}$ as a function of $p$ and $\mu_{I}$. We get

$$
\mu_{N}(s)=\frac{\frac{f(s)}{1-F(b \lambda)}-p \mu_{I}(s)}{1-p}
$$

and we can optimize over $p$ and $\mu_{I}(s)$ only subject to $\mu_{N}(s)$ being non-negative. We now show that the solution must be bang-bang, i.e. either $\mu_{I}(s)=0$ or $\mu_{N}(s)=0$ and $\mu_{I}(s)=$ $\frac{1}{p} \frac{f(s)}{1-F(b \lambda)}$.

Lemma 7. If $s \geq \lambda$, then $\mu_{I}(s)=\frac{1}{p} \frac{f(s)}{1-F(b \lambda)}$ at the optimal solution.

Proof. Take a candidate optimal policy $\left\{p, \mu_{I}().\right\}$ and suppose the set

$$
\mathcal{S}=\left\{s \geq \lambda: \mu_{I}(s)<\frac{1}{p} \frac{f(s)}{1-F(b \lambda)}\right\}
$$

has positive Lebesgue measure. We show that there exists an alternative policy where we set $\mu_{N}(s)=0$ on $\mathcal{S}$ and where we can increase $p$ without violating any constraints. Specifically, we choose $\tilde{p}>p$ and

$$
\tilde{\mu}_{I}(s)=\left\{\begin{array}{cc}
\frac{1}{\tilde{p}} \frac{f(s)}{1-F(b \lambda)} & \text { for } \quad s \in \mathcal{S} \\
\min \left\{\mu_{I}(s), \frac{1}{\tilde{p}} \frac{f(s)}{1-F(b \lambda)}\right\} & \text { for } \quad s \notin \mathcal{S}
\end{array}\right.
$$


such that $\int_{b \lambda}^{1} \tilde{\mu}_{I}(s)=1 .^{21}$ The value to the customer under this new policy is

$$
\begin{aligned}
& (1-F(b \lambda)) \tilde{p}\left(r\left(1-\int_{b \lambda}^{\lambda} \tilde{\mu}_{I}(s) d s\right)-l \int_{b \lambda}^{\lambda} \tilde{\mu}_{I}(s) d s-\alpha_{c}\right) \\
& =(1-F(b \lambda)) \tilde{p}\left(r-(r+l) \int_{b \lambda}^{\lambda} \tilde{\mu}_{I}(s) d s-\alpha_{c}\right) .
\end{aligned}
$$

His value under the old policy is

$$
(1-F(b \lambda)) p\left(r-(r+l) \int_{b \lambda}^{\lambda} \mu_{I}(s) d s-\alpha_{c}\right)
$$

which is smaller because $\tilde{p}>p$ and $\tilde{\mu}_{I}(s) \leq \mu_{I}(s)$ for $s \in[b \lambda, \lambda]$.

Lemma 8. If $s \in[b \lambda, \lambda]$, then without loss of generality $\mu_{I}(s) \in\left\{0, \frac{1}{p} \frac{f(s)}{1-F(b \lambda)}\right\}$ at the optimal solution.

Proof. Take again a candidate optimal policy such that

$$
\mathcal{S}=\left\{s \in[b \lambda, \lambda]: 0<\mu_{I}(s)<\frac{1}{p} \frac{f(s)}{1-F(b \lambda)}\right\}
$$

has positive Lebesgue measure. This policy can be replaced by one where $\mu_{I}$ puts weight on 0 and $\frac{1}{p} \frac{f(s)}{1-F(b \lambda)}$ only without altering the payoffs to adviser and customer. We choose a subset $\mathcal{S}_{1} \subset \mathcal{S}$ and define $\tilde{\mu}_{I}$ as

$$
\tilde{\mu}_{I}(s)=\left\{\begin{array}{cccc}
\frac{1}{p} \frac{f(s)}{1-F(b \lambda)} & \text { for } & s \in \mathcal{S}_{1} \\
0 & \text { for } & s \in \mathcal{S} \backslash \mathcal{S}_{1} \\
\mu_{I}(s) & \text { for } & s \notin \mathcal{S}
\end{array}\right.
$$

${ }^{21}$ This is always possible: The integral

$$
\int_{b \lambda}^{1} \tilde{\mu}_{I}(s) d s=\int_{\mathcal{S}} \frac{1}{\tilde{p}} \frac{f(s)}{1-F(b \lambda)} d s+\int_{[b \lambda, 1] \backslash \mathcal{S}} \min \left\{\mu_{I}(s), \frac{1}{\tilde{p}} \frac{f(s)}{1-F(b \lambda)}\right\} d s
$$

is decreasing in $\tilde{p}$ and as $\tilde{p} \rightarrow 1$, it is bounded by $\int_{b \lambda}^{1} \frac{f(s)}{1-F(b \lambda)} d s$ from above, which integrates to one. Since we are restricting attention to the case when $U(p)>u^{0}(b, l)$, our initial $p$ is strictly below one, so increasing $\tilde{p}>p$ until $\tilde{\mu}_{I}$ integrates to one is always feasible. 
while $p$ stays the same. $\tilde{\mu}_{I}$ satisfies

$$
\int_{b \lambda}^{1} \tilde{\mu}_{I}(s) d s=\int_{\mathcal{S}_{1}} \frac{1}{p} \frac{f(s)}{1-F(b \lambda)} d s+\int_{[b \lambda, 1] \backslash \mathcal{S}} \mu_{I}(s) d s .
$$

For any $\mu_{I}$ and $p$, we can choose $\mathcal{S}_{1} \subset \mathcal{S}$ such that $\tilde{\mu}_{I}$ integrates to one, which happens whenever ${ }^{22}$

$$
\int_{\mathcal{S}_{1}} \frac{f(s)}{1-F(b \lambda)} d s-p \int_{\mathcal{S}} \mu_{I}(s) d s=0 .
$$

The customer's value under this policy is

$$
\begin{aligned}
& (1-F(b \lambda)) p\left(r-(r+l) \int_{b \lambda}^{\lambda} \tilde{\mu}_{I}(s) d s-\alpha_{c}\right)= \\
& (1-F(b \lambda)) p\left(r-(r+l)\left(\int_{\mathcal{S}_{1}} \frac{1}{p} \frac{f(s)}{1-F(b \lambda)} d s+\int_{[b \lambda, \lambda] \backslash \mathcal{S}} \mu_{I}(s) d s\right)-\alpha_{c}\right)= \\
& (1-F(b \lambda)) p\left(r-(r+l)\left(\int_{\mathcal{S}} \mu_{I}(s) d s+\int_{[b \lambda, \lambda] \backslash \mathcal{S}} \mu_{I}(s) d s\right)-\alpha_{c}\right)= \\
& (1-F(b \lambda)) p\left(r-(r+l) \int_{b \lambda}^{\lambda} \mu_{I}(s) d s-\alpha_{c}\right),
\end{aligned}
$$

so his value remains unchanged.

Finding the optimal $\mu_{I}$ reduces to finding the optimal set $\mathcal{S}^{*} \subset[b \lambda, 1]$ so that

$$
\mu_{I}(s)=\left\{\begin{array}{ccc}
\frac{1}{p} \frac{f(s)}{1-F(b \lambda)} & \text { for } & s \in \mathcal{S}^{*} \\
0 & \text { for } & s \notin \mathcal{S}^{*}
\end{array}\right.
$$

\footnotetext{
${ }^{22}$ Since $\frac{1}{p} \frac{f(s)}{1-F(b \lambda)}>\mu_{I}(s)$ on $\mathcal{S}$, for $\mathcal{S}_{1}=\mathcal{S}$, the expression is strictly positive, while for $\mathcal{S}_{1}=\emptyset$, it is negative. The first integral in the above equation is simply $\mu_{1}\left(\mathcal{S}_{1}\right)$, i.e. the measure of $\mathcal{S}_{1}$ under $\mu_{1}$. Since $\mathcal{S}$ has positive Lebesgue measure, Lebesgue measure is atomless, and $\mu_{1}$ is absolutely continuous with respect to the Lebesgue measure, Aliprantis and Border (2006), Th. 10.52, p. 395, implies that for any number $0<q<\mu_{1}(\mathcal{S})$, there exists a measurable subset $\mathcal{S}_{1} \subset \mathcal{S}$ such that $\mu_{1}\left(\mathcal{S}_{1}\right)=q$. This establishes that we can always pick a set $\mathcal{S}_{1}$ to make $\tilde{\mu}_{I}$ integrate to one.
} 
For such a policy, the ex-ante value of the customer becomes

$$
\begin{aligned}
U & =(1-F(b \lambda)) p\left(r \int_{[\lambda, 1] \cap \mathcal{S}^{*}} \frac{1}{p} \frac{f(s)}{1-F(b \lambda)} d s-l \int_{[b \lambda, \lambda] \cap \mathcal{S}^{*}} \frac{1}{p} \frac{f(s)}{1-F(b \lambda)} d s-\alpha_{c}\right) \\
& =r \int_{[\lambda, 1] \cap \mathcal{S}^{*}} f(s) d s-l \int_{[b \lambda, \lambda] \cap \mathcal{S}^{*}} f(s) d s-\alpha_{c}(1-F(b \lambda)) \\
& =\int_{\mathcal{S}^{*}}\left(y(s, l)-\alpha_{c}\right) f(s) d s .
\end{aligned}
$$

Since $\mu_{I}$ integrates to one, we have

$$
p=\frac{1}{1-F(b \lambda)} \int_{\mathcal{S}^{*}} f(s) d s
$$

With these expressions, we can reduce the adviser's problem in (26) to

$$
\begin{aligned}
v(b, l, \bar{u}) & =\max _{\mathcal{S}^{*} \subset[b \lambda, 1]} \int_{S^{*}} f(s) d s \\
\text { s.t. } & \int_{\mathcal{S}^{*}}\left(y(s, l)-\alpha_{c}\right) f(s) d s-u^{0}(b, l) \geq \bar{u} .
\end{aligned}
$$

The problem above is analogous to choosing the acceptance region of a statistical test to maximize its power. We can use the Neyman-Peason Lemma, ${ }^{23}$ which guarantees that the optimal solution is of the form

$$
\begin{aligned}
& 1 \geq k y(s, l) \text { for } s \in \mathcal{S}^{*} \\
& 1 \leq k y(s, l) \text { for } s \notin \mathcal{S}^{*}
\end{aligned}
$$

for some constant $k \in \mathbb{R}$. For $s \in[b \lambda, \lambda] \cap \mathcal{S}^{*}$, we have $1 \geq-k l$ and for $s \in[b \lambda, \lambda] \backslash \mathcal{S}^{*}$, we have $1 \leq-k l$, so that $k=-\frac{1}{l} \cdot{ }^{24}$ Since $y(s, l)$ is piecewise constant, we can without loss of generality choose a set of the form

$$
\mathcal{S}^{*}=[\hat{s}, 1]
$$

where $\hat{s} \in[b \lambda, \lambda]$. This establishes Proposition 1 .

\footnotetext{
${ }^{23}$ See e.g. Dantzig and Wald (1951).

${ }^{24}$ The first condition becomes $1 \geq-\frac{r}{l}$ for $s \in \mathcal{S}^{*}$, which holds.
} 


\section{A.3 Proof for Lemma 2}

Consider $l^{\prime}>l$, and $b^{\prime}>b$. Then, we have

$$
\begin{aligned}
& v\left(b, l^{\prime}, U(b)\right)-v\left(b^{\prime}, l^{\prime}, U\left(b^{\prime}\right)\right) \\
= & v(b, l, U(b))-v\left(b^{\prime}, l, U\left(b^{\prime}\right)\right) \\
& -\alpha\left\{\left\{\frac{F(b \lambda) l^{\prime}}{l^{\prime}+\alpha_{c}}-\frac{F(b \lambda) l}{l+\alpha_{c}}\right\}-\left\{\frac{F\left(b^{\prime} \lambda\right) l^{\prime}}{l^{\prime}+\alpha_{c}}-\frac{F\left(b^{\prime} \lambda\right) l}{l+\alpha_{c}}\right\}\right. \\
& \left.+\left\{\frac{U(b)+\alpha_{c}}{l^{\prime}+\alpha_{c}}-\frac{U(b)+\alpha_{c}}{l+\alpha_{c}}\right\}-\left\{\frac{U\left(b^{\prime}\right)+\alpha_{c}}{l^{\prime}+\alpha_{c}}-\frac{U\left(b^{\prime}\right)+\alpha_{c}}{l+\alpha_{c}}\right\}\right\} \\
= & v(b, l, U(b))-v\left(b^{\prime}, l, U\left(b^{\prime}\right)\right) \\
& -\alpha\left\{\left(F(b \lambda)-F\left(b^{\prime} \lambda\right)\right)\left\{\frac{l^{\prime}}{l^{\prime}+\alpha_{c}}-\frac{l}{l+\alpha_{c}}\right\}+\left(U(b)-U\left(b^{\prime}\right)\right)\left\{\frac{1}{l^{\prime}+\alpha_{c}}-\frac{1}{l+\alpha_{c}}\right\}\right\} \\
> & v(b, l, U(b))-v\left(b^{\prime}, l, U\left(b^{\prime}\right)\right) .
\end{aligned}
$$

That last inequality follows from the last term is positive, given that, $l^{\prime}>l, U(b)-U\left(b^{\prime}\right)>0$, and $F(b \lambda)-F\left(b^{\prime} \lambda\right)<0$

Hence, if $l$ weakly prefer a lower $b$, then $l^{\prime}$ must be strictly prefer $b$. That is, if $v(b, l, U(b)) \geq$ $v\left(b^{\prime}, l, U\left(b^{\prime}\right)\right)$, then $v\left(b, l^{\prime}, U(b)\right)>v\left(b^{\prime}, l^{\prime}, U\left(b^{\prime}\right)\right)$.

Similarly,

$$
\begin{aligned}
& u\left(b, l^{\prime}, V\left(l^{\prime}\right)\right)-u(b, l, V(l)) \\
= & u\left(b^{\prime}, l^{\prime}, V\left(l^{\prime}\right)-u\left(b^{\prime}, l, V(l)\right)+\left(F\left(b^{\prime} \lambda\right)-F(b \lambda)\right)\left(l^{\prime}-l\right)\right. \\
> & u\left(b^{\prime}, l^{\prime}, V\left(l^{\prime}\right)-u\left(b^{\prime}, l, V(l)\right)\right.
\end{aligned}
$$

Hence, if $b^{\prime}$ weakly prefer a higher $l^{\prime}$, then $b$ must strictly prefer $l^{\prime}$.

\section{A.4 Proof for Proposition 2}

Given the constructed $\ell(b)$ and $s^{*}(b), U(b)$ is well defined. Advisers then choose which customer to attract, taking $U(b)$ as given. 


$$
\begin{aligned}
V(l) & =\max _{\tilde{b}} v(\tilde{b}, l, U(\tilde{b})) \\
& =\max _{\tilde{b}}\left(\alpha_{0}+\alpha_{c}\right)\left(1-\left(\frac{F(\tilde{b} \lambda) l}{l+\alpha_{c}}+\frac{U(\tilde{b})+\alpha_{c}}{l+\alpha_{c}}\right)\right) .
\end{aligned}
$$

The construction of $U(b)$ guarantees the FOC of advisers is satisfied, as $U^{\prime}(b)=F^{\prime}(b \lambda) \lambda \ell(b)$.

$$
\frac{d v(b, l, U(b))}{d b}=-\left(\alpha_{0}+\alpha_{c}\right)\left(\frac{F^{\prime}(b \lambda) \lambda l+U^{\prime}(b)}{\left(l+\alpha_{c}\right)}\right)
$$

The SOC is also satisfied by construction:

$$
\begin{aligned}
\left.\frac{d^{2} v(b, l, U(b))}{d^{2} b}\right|_{b=\ell^{-1}(l)} & =-\frac{\left(\alpha_{0}+\alpha_{c}\right)}{\left(l+\alpha_{c}\right)}\left(\left(F^{\prime \prime}\left(\ell^{-1}(l) \lambda\right) \lambda^{2} l+U^{\prime \prime}\left(\ell^{-1}(l)\right)\right)\right. \\
& =\frac{\left(\alpha_{0}+\alpha_{c}\right)}{\left(l+\alpha_{c}\right)} F^{\prime}\left(\ell^{-1}(l) \lambda\right) \ell^{\prime}(b)<0
\end{aligned}
$$

where as $U^{\prime}(b)=-F^{\prime}(b \lambda) \lambda \ell(b)$ and $U^{\prime \prime}(b)=-F^{\prime \prime}(b \lambda) \lambda^{2} \ell(b)-F^{\prime}(b \lambda) \lambda \ell^{\prime}(b)$. This thus proves the optimality of advisers (E2).

Customers' optimization problem:

$$
\begin{aligned}
U(b) & =\max _{l}\left\{\tilde{U}\left(b, l, s^{*}(l)\right)-u^{0}(b, l)\right\} \\
& =\max _{l}\left(F\left(s^{*}(l)\right)-F(b \lambda)\right) l-\alpha_{c}\left(1-F\left(s^{*}(l)\right)\right)
\end{aligned}
$$

The ODE of $s^{*}(l)(22)$ guarantees the FOC of (21) is satisfied.

$$
\frac{d\left\{\tilde{U}\left(b, l, s^{*}(l)\right)-u^{0}(b, l)\right)}{d l}=\left(F\left(s^{*}(l)\right)-F(b \lambda)\right)+F^{\prime}\left(s^{*}(l)\right)\left(l+\alpha_{c}\right) \frac{d s^{*}(l)}{d l}
$$

The second order condition yields

$$
\left.\frac{d^{2}\left\{\tilde{U}\left(b, l, s^{*}(l)\right)-u^{0}(b, l)\right)}{d^{2} l}\right|_{l=\ell(b)}=F^{\prime}\left(\ell^{-1}(l) \lambda\right) \frac{d \ell^{-1}(l)}{d l}=F^{\prime}(b \lambda) \lambda \frac{1}{\ell^{\prime}(b)}<0
$$


This thus proves customers' optimalitiy (E1).

\section{A.5 Proof of Lemma 4}

The information policy must solved the following equation:

$$
\frac{d s^{*}(l)}{d l}=-\frac{\left(F\left(s^{*}(l)\right)-F(\beta(l) \lambda)\right.}{F^{\prime}\left(s^{*}(l)\right)(l+\alpha)} \equiv m(l, \alpha) .
$$

When $Q(\bar{b})<1$, the initial condition is then given by the full disclosure: $s_{0}^{* \alpha^{\prime}}=s_{0}^{* \alpha}=\lambda$. When $Q(\bar{b})>1$, the initial condition $s^{* \alpha}\left(l^{*}\right)=s_{0}^{* \alpha}$ increases with $\alpha$. Hence, overall $s_{0}^{* \alpha^{\prime}} \geq$ $s_{0}^{* \alpha^{\prime}}$, together with $m\left(l, \alpha^{\prime}\right)>m(l, \alpha)$, by comparison theorem: $s^{*}\left(l, \alpha^{\prime}\right)>s^{*}(l, \alpha)$.

\section{B Advisers as Gatekeepers}

In our main model, in order to isolate the value of information provided by advisers, we assumed that customers can always purchase the asset even if they do not receive advice. However, in many environments advisers act as gatekeepers. Medical procedures cannot be accessed without first receiving a doctor's advice, prescription drugs cannot be bought without consultation from a pharmacist, and, although not strictly mandated, winning a complex trial without consulting a lawyer is effectively impossible.

We will retain the language of customers, advisers, and assets from our basic setup. The key difference is that without requesting advice, the customer's value for any asset is zero, instead of his value from deciding based on his own signal. As we show now, this has profound effects. Sorting between customers and advisers disappears, so that every customer is indifferent between requesting advice for any asset. In the pure advice case, advisers with more information sensitive assets were providing worse information, which earned them higher rents. Here, the opposite is true. Since a higher $l$ asset has lower ex-ante value, advisers must compensate customers with providing better information, which lowers their profits. Thus, when advisers act as gatekeepers, the key findings from the pure advice case are reversed.

To be concrete, we assume that all customers have access to a default option with payoff $\left(r^{0}, l^{0}\right)$. We further assume that the default option is the least information sensitive so that $l^{0}<l$ and $\forall l$ and $r^{0}<r$. 
We further impose the following parameter restriction so that the role of information is more interesting:

Assumption 1. (A3) Without any further information, the default option has a higher expected return: $(1-F(\lambda))\left(r-r^{0}\right)-F(\lambda)\left(l-l^{0}\right)<0$. (A4) If full information is being provided, the default option is dominated: $(1-F(\lambda)) r>(1-F(\lambda)) r^{0}-(F(\lambda)-F(\bar{b} \lambda)) l^{0}$.

(A3) guarantees that in order to attract customers, advisers must provide at least some information. (A4) ensures that in equilibrium, customers don't always choose the default option.

Customer $b$ 's outside option is then given by

$$
u^{0}(b)=\left(1-F(\lambda) r^{0}-\left((F(\lambda)-F(b \lambda)) l^{0},\right.\right.
$$

which is increasing in $b$ as the more informed customers can always do better.

In order to invest in asset $l$, customers must match with adviser $l$. The optimization problem of an adviser is given by,

$$
\begin{aligned}
& v(a, b, u) \equiv \max _{s \in[b \lambda, \lambda]}(1-F(s)) \alpha \\
& \text { s.t. }(1-F(\lambda)) r-(F(\lambda)-F(s)) l-\alpha_{c}(1-F(s)) \geq u .
\end{aligned}
$$

The LHS of the constraint represents the expected payoff of customer under policy $\hat{s}$. Note that, given that customers are not allowed to invest asset $l$ themselves, they will simply choose the adviser that provides the highest payoff. On the other hand, in our main model, customers will choose the adviser that provides the highest information gains. The solution yields

$$
v(l, b, u)=\alpha\left(1-\left(F(\lambda)+\frac{u+\alpha_{c}-(1-F(\lambda)) r}{l+\alpha_{c}}\right)\right) .
$$

From the solution, we can see two important differences: First,

$$
v_{l}=\frac{u+\alpha_{c}-(1-F(\lambda)) r}{\left(l+\alpha_{c}\right)^{2}}<0 .
$$

Hence, in equilibrium, advisers with higher $l$ must receive a lower payoff. This is because selecting advisers here also means selecting assets. Since a higher $l$ has a lower expected 
return (thus is a worse asset), it is harder to attract customers. Thus, these advisers receive lower payoff. ${ }^{25}$

Second, conditional on giving utility $u$ to a customer, an adviser now does not care about which customer to attract. This implies that all customers must have the same $u$ conditional on being active in equilibrium. Therefore, there is no sorting among customers and advisers.

Without sorting, all active customers must be indifferent between different assets in equilibrium. As a result, if trading an asset with higher loss $l$, customers must be compensated with better information. That is, for any active $b$ and $l$, the following condition must hold:

$$
\tilde{U}\left(b, l, s^{*}(l)\right)=(1-F(\lambda)) r-\left(F(\lambda)-F\left(s^{*}(l)\right)\right) l-\alpha_{c}\left(1-F\left(s^{*}(l)\right)\right)=U^{*} .
$$

The above equation yields the ODE which pins down $s^{*}(l)$ :

$$
\frac{d s^{*}(l)}{d l}=\frac{F(\lambda)-F\left(s^{*}(l)\right)}{\left(l+\alpha_{c}\right) F^{\prime}\left(s^{*}(l)\right)}>0 .
$$

That is, an adviser with a higher $l$ must give away more precise information, thus receiving a lower payoff.

Proposition 4 (Advisers as gatekeepers). Under (A3) and (A4), the equilibrium is characterized by a marginal participant and policy function $s^{*}(l)$. All actives advisers and customers are indifferent among whom to match with.

(1) When $Q(\bar{b})>1$, there exists a marginal customer $b^{*}=Q^{-1}(1)$ such that only customers with $b \leq b^{*}$ participate the market. The information policy $s^{*}(l)$ solves equation (29) and the initial condition $s^{*}(\bar{l})$ solves $\tilde{U}\left(b, \bar{l}, s^{*}(\bar{l})\right)=u\left(b^{*}\right)$.

(2) When $Q(\bar{b})<1$, there exists a marginal adviser $l^{*}$, which solves $G\left(l^{*}\right)=Q(\bar{b})$. Only advisers with lower $l \leq l^{*}$ participate the market. The information policy $s^{*}(l)$ solves equation (29) with the initial condition $s^{*}\left(l^{*}\right)=\lambda$.

Since there is no sorting, the equilibrium is pinned by the marginal type. As before, a less informed customer always has higher incentive to participate. Hence, when advisers are on the short side of the market, the information provided by the worst asset must be such that the marginal customer is indifferent, which explains Result (1).

\footnotetext{
${ }^{25}$ On the other hand, our main model looks at the value of information only, so selecting advisers equals selecting the most valuable information only. Thus, $v_{l}>0$ which is then increasing in $l$.
} 


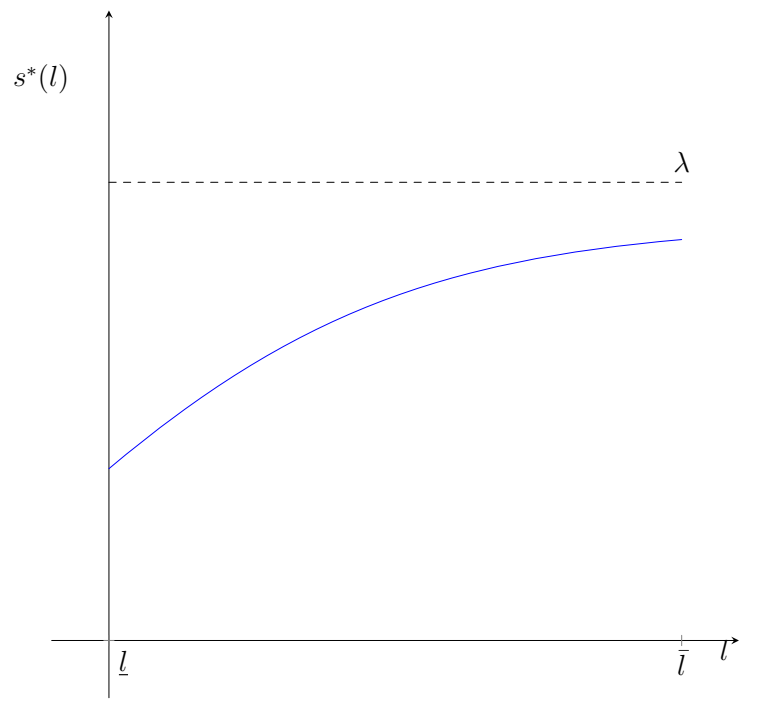

Figure 4: Information Policy with Scarce Advisers

On the other hand, when customers are on the short side, advisers with relatively higher $l$ must be the ones being unmatched, as they are the one who must gain less in equilibrium. Such a marginal adviser $l^{*}$ must give his customers the highest payoff possible, meaning full disclosure for the worst active assets.

The equilibrium information policy when advice is scarce is illustrated in Figure 4. 


\section{References}

Aliprantis, C. D. and K. Border (2006). Infinite dimensional analysis: a hitchhiker's guide. Springer Science \& Business Media.

Anagol, S., S. A. Cole, and S. Sarkar (2013). Understanding the advice of commissionsmotivated agents: Evidence from the Indian life insurance market. Harvard Business School Finance Working Paper (12-055).

Au, P. H. and K. Kawai (2015). Competition in Information Disclosure. Available at SSRN 2705326.

Becker, G. S. (1973). A theory of marriage: Part i. The Journal of Political Economy, $813-846$.

Bergstresser, D., J. M. Chalmers, and P. Tufano (2009). Assessing the costs and benefits of brokers in the mutual fund industry. Review of financial studies 22(10), 4129-4156.

Board, S. and J. Lu (2015). Competitive information disclosure in search markets. Unpublished.

CEA (2015). The Effects of Conflicted Investment Advice on Retirement Savings. Technical report, Executive Office of the President.

Chalmers, J. and J. Reuter (2010). What is the Impact of Financial Advisors on Retirement Portfolio Choices \& Outcomes? Technical report, National Bureau of Economic Research.

Chiappori, P.-A. and P. Reny (2006). Matching to share risk. manuscript http://home. uchicago. edu/ ${ }^{\sim}$ preny/papers/matching-05-05-06. pdf.

Dantzig, G. B. and A. Wald (1951). On the fundamental lemma of Neyman and Pearson. The Annals of Mathematical Statistics 22(1), 87-93.

Gentzkow, M. and E. Kamenica (2011). Competition in persuasion. Technical report, National Bureau of Economic Research.

Hoechle, D., S. Ruenzi, N. Schaub, and M. Schmid (2013). Don't answer the phone: financial advice and individual investors' performance. Technical report, Working paper.

Inderst, R. and M. Ottaviani (2009). Misselling through agents. The American Economic Review $99(3)$, 883-908. 
Inderst, R. and M. Ottaviani (2012a). Competition through commissions and kickbacks. The American Economic Review 102(2), 780-809.

Inderst, R. and M. Ottaviani (2012b). How (not) to pay for advice: A framework for consumer financial protection. Journal of Financial Economics 105(2), 393-411.

Kamenica, E. and M. Gentzkow (2011). Bayesian persuasion. The American Economic Review 101(6), 2590-2615.

Legros, P. and A. F. Newman (2007). Beauty is a beast, frog is a prince: assortative matching with nontransferabilities. Econometrica 75 (4), 1073-1102.

Li, F. and P. Norman (2015). On Bayesian persuasion with multiple senders. Available at SSRN 250527\%.

Linnainmaa, J. T., B. T. Melzer, A. Previtero, and C. Grace (2015). Costly Financial Advice: Conflicts of Interest or Misguided Beliefs? December.

Mullainathan, S., M. Noeth, and A. Schoar (2012). The market for financial advice: An audit study. Technical report, National Bureau of Economic Research.

Ostrovsky, M. and M. Schwarz (2010). Information disclosure and unraveling in matching markets. American Economic Journal: Microeconomics 2(2), 34-63.

Shapley, L. S. and M. Shubik (1971). The assignment game i: The core. International Journal of game theory 1(1), 111-130.

Terviö, M. (2008). The difference that CEOs make: An assignment model approach. The American Economic Review 98(3), 642-668. 